\title{
Proteome characterization of developing grains in bread wheat cultivars (Triticum aestivum L.)
}

\author{
Guangfang Guo ${ }^{\dagger}$, Dongwen $\mathrm{LV}^{\dagger}$, Xing Yan`, Saminathan Subburaj, Pei Ge, Xiaohui Li, Yingkao Hu
} and Yueming Yan

\begin{abstract}
Background: The analyses of protein synthesis, accumulation and regulation during grain development in wheat are more complex because of its larger genome size compared to model plants such as Arabidopsis and rice. In this study, grains from two wheat cultivars Jimai 20 and Zhoumai 16 with different gluten quality properties were harvested at five development stages, and were used to displayed variable expression patterns of grain proteins.

Results: Proteome characterization during grain development in Chinese bread wheat cultivars Jimai 20 and Zhoumai 16 with different quality properties was investigated by 2-DE and tandem MALDI-TOF/TOF-MS. Identification of 117 differentially accumulated protein spots representing 82 unique proteins and five main expression patterns enabled a chronological description of wheat grain formation. Significant proteome expression differences between the two cultivars were found; these included 14 protein spots that accumulated in both cultivars but with different patterns and 27 cultivar-different spots. Among the cultivar-different protein spots, 14 accumulated in higher abundance in Jimai 20 than in Zhoumai 16, and included NAD-dependent isocitrate dehydrogenase, triticin precursor, LMW-s glutenin subunit and replication factor C-like protein. These proteins are likely to be associated with superior gluten quality. In addition, some proteins such as class II chitinase and peroxidase 1 with isoforms in developing grains were shown to be phosphorylated by Pro-Q Diamond staining and phosphorprotein site prediction. Phosphorylation could have important roles in wheat grain development. qRT-PCR analysis demonstrated that transcriptional and translational expression patterns of many genes were significantly different.

Conclusions: Wheat grain proteins displayed variable expression patterns at different developmental stages and a considerable number of protein spots showed differential accumulation between two cultivars. Differences in seed storage proteins were considered to be related to different quality performance of the flour from these wheat cultivars. Some proteins with isoforms were phosphorylated, and this may reflect their importance in grain development. Our results provide new insights into proteome characterization during grain development in different wheat genotypes.
\end{abstract}

Keywords: Wheat, Grain proteome, Phosphorproteins, 2-DE, Tandem MS, qRT-PCR

\section{Background}

Wheat is one of the three most important crops in the world due to its value as a major food source and its unique suitability to bread production. The protein composition of wheat grain is the key to bread baking quality. Wheat grain proteins are classified into non-prolamins, including albumins and globulins, and prolamins including

\footnotetext{
* Correspondence: yanym@mail.cnu.edu.cn

${ }^{\dagger}$ Equal contributors

College of Life Science, Capital Normal University, Beijing 100048, China
}

gliadins and glutenins [1,2]. Albumins and globulins are more abundant in essential amino acids such as lysine, tryptophan and methionine that are very important for human health. Prolamins are the major storage proteins and determine the viscoelasticity of dough. Understanding protein synthesis and regulation during grain development is of fundamental importance for targeting wheat quality by conventional breeding or genetic engineering to specific end-uses.

The development of wheat grain involves three distinct phases: cell division and differentiation, grain filling, and

\section{Biomed Central}


desiccation/maturation [3]. In recent years, studies on transcriptomics, proteomics and metabolomics have provided insights into the mechanism of reserve accumulation during wheat grain development. For instance, using Affymetrix wheat GeneChip oligonucleotide arrays, 14,550 transcripts showed significant differential regulation in developing caryopses of hexaploid wheat cv. Hereward between 6 and 42 days after anthesis [3]. Transcriptomic and metabolomic approaches have been used to investigate the impacts of nitrogen $(\mathrm{N})$ and sulphur (S) deficiency on $\mathrm{N}$ and $\mathrm{S}$ remobilization during grain filling in winter wheat [4]. It has been noted, however, that the accumulated amounts of a large proportion of proteins are often poorly correlated with their corresponding mRNAs in expression profiles [5-7] and hence direct proteome approaches can be more valuable in monitoring developmental profiles. Two-dimensional electrophoresis (2-DE) and mass spectrometry (MS) proteomic approaches provide the tools for the monitoring the dynamic expression profiles of proteins during seed development, and have been widely used in different plant species such as Arabidopsis [5,8], Medicago truncatula [9], soybean [10], maize [11] and rice [12,13]. Considerable work on wheat grain proteomics has been carried out in different wheat varieties $[14,15]$, endosperm and endosperm amyloplasts [16,17], kernel aleurone and peripheral layers [18], and grain storage proteins [19-21]. Recent work has focused on proteomic analysis of different grain developing stages, such as six grain developmental stages in winter wheat variety Récital [22], five developmental stages in Jing 411 and Sunstate at [23], four developmental stages in two near-isogenic lines of bread wheat cv. Falcon [24], four developmental stages in two spring wheat varieties Ningchun 4 and Chinese Spring under drought stress [25]. Although these studies provided valuable information, the biochemical processes important for wheat grain development still require research because this species has a much larger genome and a more complex proteome than model plants such as Arabidopsis and rice.

A complex gene network regulates protein expression during grain development [26]. Various post-translational modifications of proteins (PTMs) occur as grains develop and mature. The poor association between transcription (mRNA) and translation (protein) levels indicates the importance of PTMs. Protein phosphorylation, as an important PTM and a transient and reversible modification, plays a crucial role in signaling and regulation of cellular processes such as proliferation, differentiation, and apoptosis [27]. For example, phosphorylation in wheat amyloplasts is capable of regulating starch branching enzyme activity and protein-protein interactions [28]. Among the many strategies for studying protein phosphorylation, a powerful way for directly identifying phosphorylated proteins is to separate phosphoproteins by 2-DE and to stain with phosphospecific dyes such as Pro-Q Diamond phosphoprotein staining followed by tandem mass spectrometry analyses [29-31].

In the present work, we conducted an investigation on proteome characterization of developing grains in two bread wheat cultivars (Jimai 20 and Zhoumai 16) with different gluten quality properties, by 2-DE and MALDITOF/TOF-MS. Jimai 20 has high yield and superior gluten quality, whereas Zhoumai 16 has poor gluten quality and higher yield [32]. In the past ten years, both cultivars were widely cultivated in the main wheat areas of China. Our results provide a comprehensive view of proteome characterization during grain development in different wheat genotypes.

\section{Results}

\section{Grain development and SEM observations}

In general, grain size and weight in both Jimai 20 and Zhoumai 16 increased gradually from flowering to maturity, but their development rates and grain sizes were different (Figure 1A, B). Zhoumai 16 had a larger grain size and higher grain weight than Jimai 20 at all grain developmental stages except the first. SEM observations on both cultivars indicated that starch granules accumulated continuously until grain maturity (Figure 1C). As previously observed [33,34], A (diameter $>10 \mu \mathrm{m})$ and B (diameter $<10 \mu \mathrm{m}$ ) starch granules appeared at $6 \mathrm{DPA}$ $\left(147^{\circ} \mathrm{Cd}\right)$ and $11 \mathrm{DPA}\left(252^{\circ} \mathrm{Cd}\right)$, respectively. The size of A granules as well as grain weight increased rapidly from 11 to $15 \mathrm{DPA}\left(252-353^{\circ} \mathrm{Cd}\right)$, but $\mathrm{B}$ granules grew only slowly from 11 to $31 \mathrm{DPA}$. This indicated that the period 11-15 DPA was a key stage for grain starch synthesis and accumulation.

\section{Identification, classification and localization of differentially accumulated proteins during grain development in the two cultivars}

Grain proteins extracted at the five developmental stages in the two cultivars were separated by 2-DE with broadrange ( $\mathrm{pH}$ 3-10 L) IPG strips. The proteome profiles were generally similar in both cultivars at all five stages (Figure 2) (Figure 3). Most of the proteins on the 2-DE gels were distributed in the $\mathrm{pH} 3-7$ range during the earlier (I and II) development stages. The numbers of basic proteins increased considerably from stage III, especially in the last stage. In total, 174 protein spots showed more than two fold differences in abundance, of which 117 representing 84 unique proteins were identified by MALDI-TOF/TOF-MS (Table 1). Their peptide sequences are listed in Additional file 1: Table S1.

According to their functions, the identified proteins were classified into several main groups, including carbohydrate 


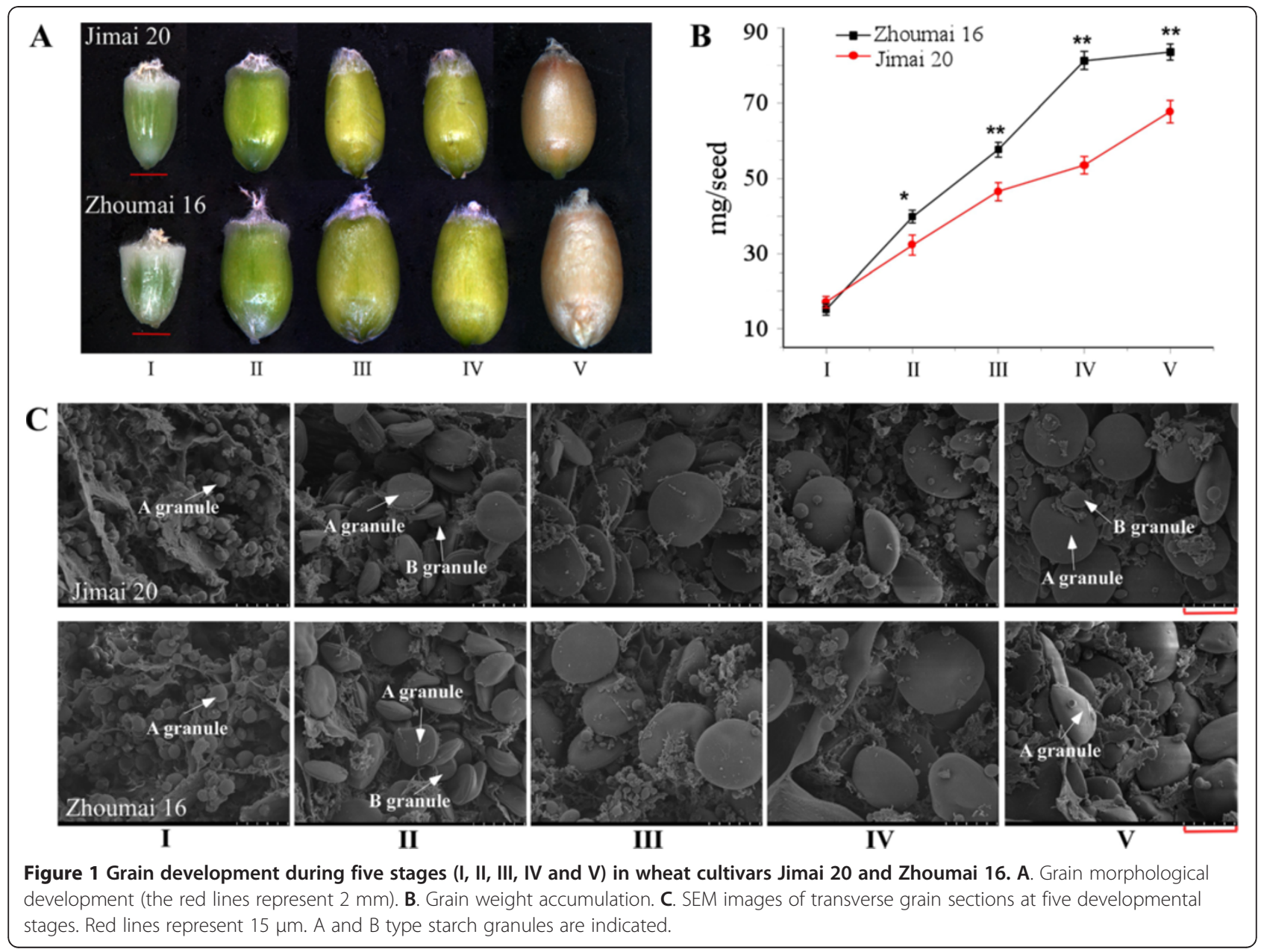

metabolism (31.6\%), stress/defense (25.6\%), storage proteins (14.5\%), protein synthesis/assembly/degradation (7.7\%), transcription/translation (7.7\%), nitrogen metabolism $(5.1 \%)$ and signal transduction (3.4\%) as shown as in Figure 4. More than $80 \%$ were identified as enzymes. Proteins related to carbohydrate metabolism contained five subcategories: glycolysis (18\%), TCA pathway (5\%), lipid and sterol metabolism (3\%), starch metabolism (3\%) and alcoholic fermentation (2\%).

Among the differentially accumulated proteins, 25 different kinds were represented by two or more spots, and more than 10 isoforms were identified with different molecular masses or isoelectric points, each having two or three protein spots located at different positions on the same gel (Figure 2 and 5A), such as phosphoglycerate mutase (spots 1 and 2), glucose-1phosphate adenylyltransferase (spots 41 and 44), triticin (spots 67 and 68), alpha-amylase inhibitor CM16 subunit (spots 98 and 99), monomeric alpha-amylase inhibitor (spots 119 and 120), class II chitinase (spots 112-116) and peroxidase 1 (spots 121, 122 and 123).
Some of these isoforms might have resulted from certain PTMs such as phosphorylation.

In order to confirm the presence of phosphorylated proteins with isoforms, grain proteins at stage $\mathrm{V}$ were separated by 2-DE and then subjected to Pro-Q Diamond staining (Invitrogen) to detect putative phosphorylated proteins. Many spots stained in different intensities on the gels, indicating they were phosphorylated (Figure 5B). Sixteen spots with intense signals in both cultivars were identified by MALDI-TOF/TOF-MS (Table 2); their peptide sequences are listed in Additional file 2: Table S2. These phosphoproteins were mainly involved in stress and defense and the isoforms were particularly well stained, such as class II chitinase (gi/62465514) at spots 112, 113 and 114, and peroxidase 1 (gi/22001285) at spots 122 and 123 (Figure 5A).

Phosphorylated modification sites on serine, threonine and tyrosine were predicted by NetPhos 2.0 Serve [35,36]. Generally, the predicted results were in accordance with those from Pro-Q Diamond dye staining (Table 2). For example, class II chitinase (spots 112, 113 and 114, gi/ 

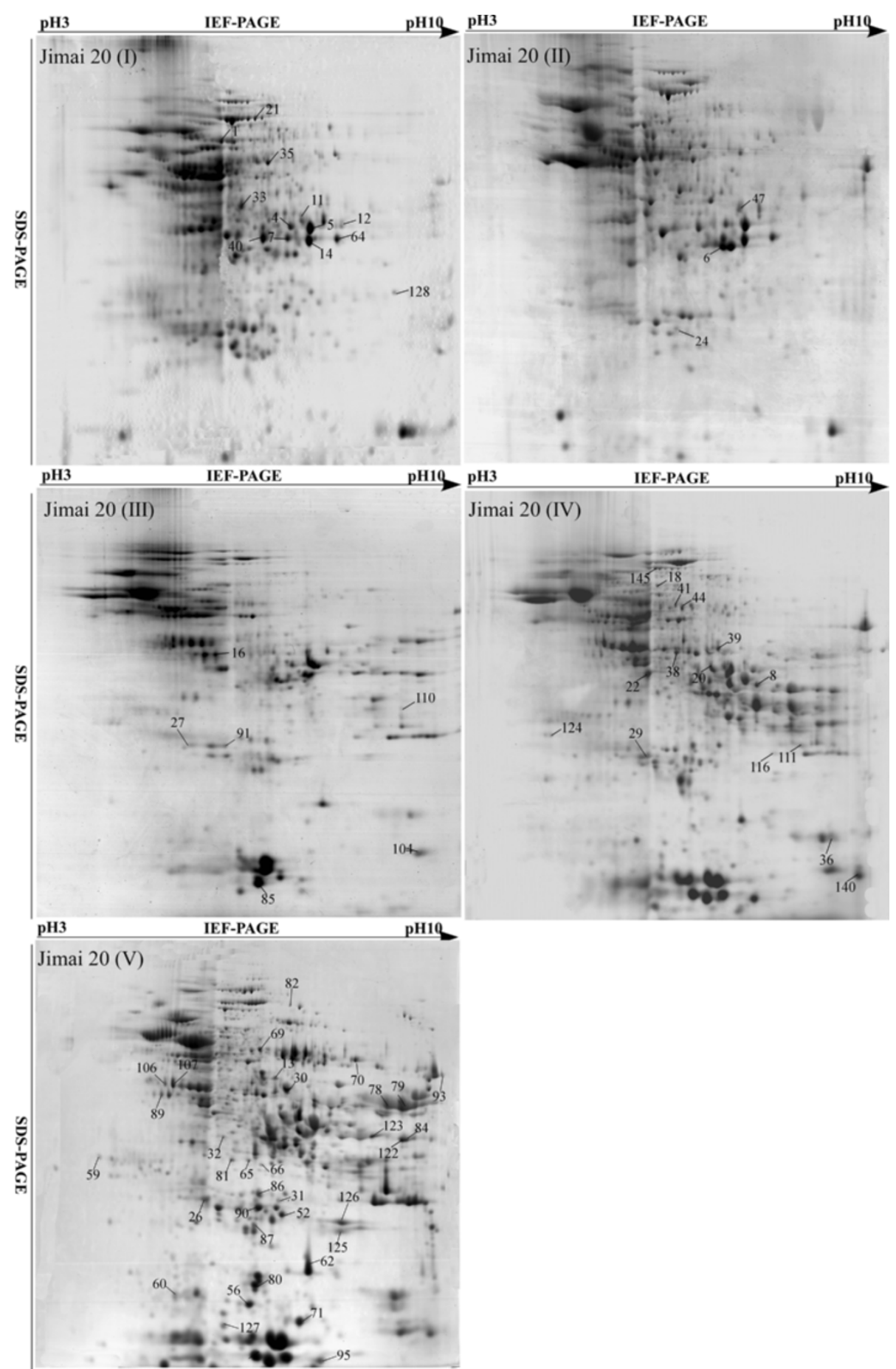

Figure 2 2-DE maps of proteins extracted from the first sample of Jimai 20.

62465514) was predicted to possess 9 serine, 3 threonine and 1 tyrosine phosphorylated modification sites, whereas peroxidase 1 (spots 122 and 123, gi/22001285) had 11, 5 and 2 phosphorylated modification sites of serine, threonine and tyrosine, respectively. Phosphorylated protein staining showed that both proteins had strong staining signals (Figure 5B).

Through sub-cellular localization of the identified proteins during grain development, a large number of proteins appeared to locate in the cytosol (Table 1). Most of the 


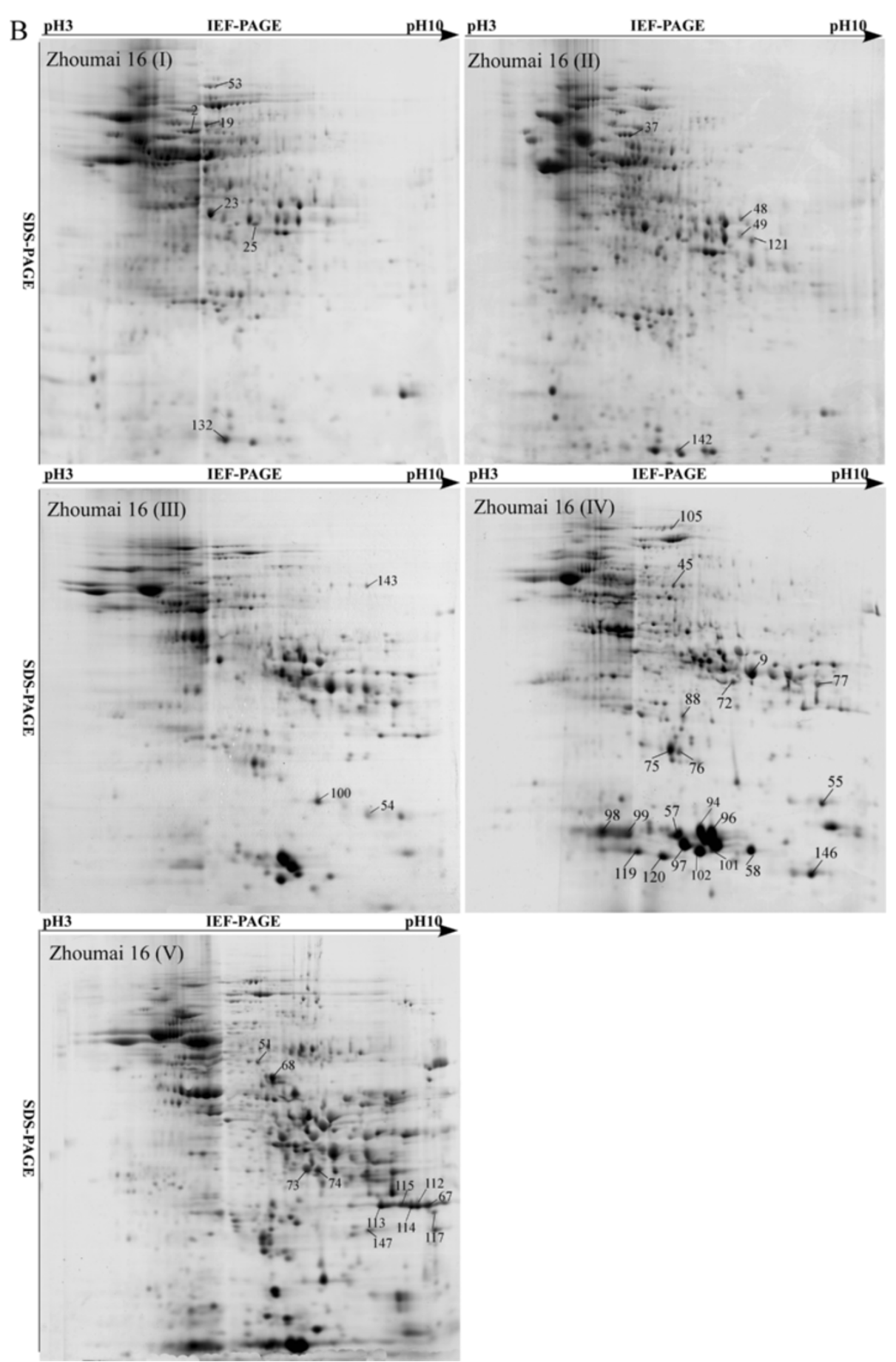

Figure 3 Zhoumai 16 at five stages of grain development.

identified enzymes with higher abundance, and involved in glycolysis-the TCA pathway-nitrogen metabolism were located in the cytosol especially during the first two stages. Until stage III many storage proteins appeared on the endoplasmic reticulum (ER). Stress-related proteins were mainly located in the plastids and were extracellular at all development stages. Additionally, some enzymes involved in starch synthesis also appeared in plastids during the early development stages.

\section{Protein expression profiles during grain development}

The expression profiles of the 117 protein spots were investigated by hierarchical cluster analysis (Figure 6). Five main expression patterns (A-E) were present and 
Table 1 Differentially expressed proteins identified by MALDI-TOF/TOF-MS at five grain developmental stages in bread wheat cultivars Jimai 20 and Zhoumai 16

\begin{tabular}{|c|c|c|c|c|c|c|c|c|c|c|c|c|c|}
\hline \multirow[t]{2}{*}{$\begin{array}{l}\text { Spot } \\
\text { no. }^{a}\end{array}$} & \multirow[t]{2}{*}{$\begin{array}{c}\text { Accession } \\
\text { no. }\end{array}$} & \multirow[t]{2}{*}{ Protein name } & \multirow[t]{2}{*}{ Species } & \multirow{2}{*}{$\begin{array}{l}\text { Protein } \\
\text { score } \\
\text { C.I. } \%^{c}\end{array}$} & \multirow{2}{*}{$\begin{array}{l}\text { Total } \\
\text { lon } \\
\text { score }\end{array}$} & \multirow[t]{2}{*}{$\mathrm{MP}^{\mathrm{d}}$} & \multirow[t]{2}{*}{$E p l / T p I^{e}$} & \multicolumn{2}{|c|}{$\begin{array}{l}\text { Average volume } \\
\text { ratio (Jimai 20) }\end{array}$} & \multicolumn{2}{|c|}{$\begin{array}{l}\text { Average volume } \\
\text { ratio (Zhoumai 16) }\end{array}$} & \multirow[t]{2}{*}{$\begin{array}{l}\text { HCP } \\
(J / Z)^{f}\end{array}$} & \multirow{2}{*}{$\begin{array}{r}\text { Predicted } \\
\text { subcellula } \\
\text { Localizatior }\end{array}$} \\
\hline & & & & & & & & I:II:III:IV:V & $p$-value & I:II:III:IV:V & $p$-value & & \\
\hline \multicolumn{14}{|c|}{ Carbohydrate metabolism } \\
\hline \multicolumn{14}{|c|}{ 1. TCA pathway: } \\
\hline 22 & gi/49343245 & Cytosolic malate dehydrogenase & T. aestivum & 100 & 161 & 6 & $5.36 / 5.75$ & 1:1.1:2.4:1.3:0.3 & 0.015 & 1:1.2:0.8:0.3:0.2 & 0.036 & $C / C$ & Cyto \\
\hline 23 & gi/49343245 & Cytosolic malate dehydrogenase & T. aestivum & 100 & 51 & 2 & $5.76 / 5.75$ & 1:1.5:2.6:1.4:0.4 & 0.023 & 1:1.4:1.0:0.4:0.3 & 0.030 & $\mathrm{C} / \mathrm{C}$ & Cyto \\
\hline 24 & gi/37928995 & Cytosolic malate dehydrogenase & T. aestivum & 100 & 266 & 3 & $5.98 / 6.62$ & 1:0.7:0.4:0.3:0 & 0.038 & 1:0.7:0.6:0:0 & 0.027 & $\mathrm{~A} / \mathrm{A}$ & Cyto \\
\hline 25 & gi/15232820 & MDH (malate dehydrogenase) & A. thaliana & 100 & 101 & 2 & $6.82 / 8.66$ & 1:0.8:0.8:0.5:0.3 & 0.019 & $1: 0.7: 1.0: 0.7: 0.4$ & 0.019 & A/A & $P$ \\
\hline 32 & $\mathrm{gi} / 7488742$ & $\begin{array}{l}\text { Isocitrate dehydrogenase } \\
\text { (NADP) precursor }\end{array}$ & M. sativa & 99 & 144 & 3 & $5.54 / 6.15$ & 1:0.6:0.7:0.5:0.1 & 0.041 & 1:0.9:0.5:0.4:0.2 & 0.011 & A/A & $P$ \\
\hline 33 & gi/92875135 & $\begin{array}{l}\text { Isocitrate dehydrogenase } \\
\text { NADP-dependent }\end{array}$ & M. truncatula & 97.38 & 98 & 2 & $6.02 / 5.99$ & 1:0.8:0.5:0.3:0.3 & 0.021 & 1:0.5:0.5:0.2:0.2 & 0.018 & A/A & Mito \\
\hline \multicolumn{14}{|c|}{ 2. Glycolysis: } \\
\hline 1 & gi/32400802 & Phosphoglycerate mutase & T. aestivum & 100 & 71 & 2 & $5.55 / 5.43$ & 1:0.4:0.4:0.2:0.1 & 0.032 & $1: 0.5: 0.3: 0.2: 0$ & 0.029 & A/A & Cyto \\
\hline 2 & gi/32400802 & Phosphoglycerate mutase & T. aestivum & 100 & 55 & 2 & $5.45 / 5.43$ & 1:0.7:0.5:0.3:0.2 & 0.017 & 1:0.6:0.6:0.5:0.3 & 0.034 & $\mathrm{~A} / \mathrm{A}$ & Cyto \\
\hline 4 & gi/120680 & $\begin{array}{l}\text { Glyceraldehyde-3-phosphate } \\
\text { dehydrogenase, cytosolic }\end{array}$ & H. vulgare & 100 & 323 & 4 & $6.46 / 6.67$ & 1:1.4:0.9:0.4:0.2 & 0.025 & 1:1.0:0.3:0.3:0.1 & 0.015 & C/A & Cyto \\
\hline 5 & gi/120680 & $\begin{array}{l}\text { Glyceraldehyde-3-phosphate } \\
\text { dehydrogenase, cytosolic }\end{array}$ & H. vulgare & 100 & 430 & 4 & $6.67 / 6.67$ & 1:1.2:0.9:0.5:0.4 & 0.014 & 1:1.5:0.8:0.5:1.1 & 0.014 & $C / C$ & Cyto \\
\hline 6 & gi/120668 & $\begin{array}{l}\text { Glyceraldehyde-3-phosphate } \\
\text { dehydrogenase, cytosolic }\end{array}$ & H. vulgare & 100 & 317 & 4 & $6.43 / 6.20$ & 1:2.1:0.5:0.8:0.9 & 0.024 & 1:1.9:1.0:0.5:0.6 & 0.022 & $C / C$ & Cyto \\
\hline 7 & gi/148508784 & $\begin{array}{l}\text { Glyceraldehyde-3-phosphate } \\
\text { dehydrogenase }\end{array}$ & T. aestivum & 100 & 99 & 4 & $6.44 / 7.08$ & 1:1.6:0.6:0.5:0.2 & 0.026 & 1:1.2:0.4:0.4:0.1 & 0.011 & $C / C$ & Cyto \\
\hline 8 & gi/148508784 & $\begin{array}{l}\text { Glyceraldehyde-3-phosphate } \\
\text { dehydrogenase }\end{array}$ & T. aestivum & 100 & 137 & 4 & 7.47/7.08 & 1:1.3:0.4:0.4:0.3 & 0.031 & - & - & $\mathrm{C} /-$ & Cyto \\
\hline 9 & gi/148508784 & $\begin{array}{l}\text { Glyceraldehyde-3-phosphate } \\
\text { dehydrogenase }\end{array}$ & T. aestivum & 100 & 126 & 2 & 7.36/7.08 & 1:1.4:3.2:5.8:0.2 & 0.037 & 1:1.3:6.2:3.2:2.4 & 0.030 & $C / C$ & Cyto \\
\hline 11 & gi/18978 & $\begin{array}{l}\text { Glyceraldehyde 3-phosphate } \\
\text { dehydrogenase }\end{array}$ & H. vulgare & 99.83 & 351 & 4 & $6.82 / 6.67$ & 1:1.3:1:0.6:0.2 & 0.011 & 1:0.9:1.7:1.1:0.1 & 0.010 & $C / C$ & Cyto \\
\hline 12 & gi/18978 & $\begin{array}{l}\text { Glyceraldehyde 3-phosphate } \\
\text { dehydrogenase }\end{array}$ & H. vulgare & 100 & 131 & 1 & $7.23 / 6.67$ & 1:0.8:0.5:0.2:0 & 0.029 & 1:0.5:0.2:0:0 & 0.024 & $\mathrm{~A} / \mathrm{A}$ & Cyto \\
\hline 13 & gi/7579064 & $\begin{array}{l}\text { Cytosolic glyceraldehyde-3- } \\
\text { phosphatedehydrogenase } \\
\text { GAPDH }\end{array}$ & T. aestivum & 100 & 238 & 3 & $6.16 / 7.83$ & $0: 0: 0: 1: 1.3$ & 0.041 & 0:0:0:1.0:1.9 & 0.011 & $B / B$ & Cyto \\
\hline 14 & gi/32478662 & $\begin{array}{l}\text { Cytosolic glyceraldehyde-3- } \\
\text { phosphatedehydrogenase }\end{array}$ & T. aestivum & 100 & 208 & 2 & $6.66 / 6.34$ & 1:1.2:0.8:0.6:0.3 & 0.005 & 1:1.4:0.8:0.5:0.5 & 0.023 & $\mathrm{C} / \mathrm{C}$ & Cyto \\
\hline 16 & gi/28172909 & $\begin{array}{l}\text { Cytosolic 3-phosphoglycerate } \\
\text { kinase }\end{array}$ & T. aestivum & 100 & 52 & 2 & $4.76 / 4.91$ & 1:1.6:2.1:1.7:0.4 & 0.019 & 1:1.9:1.6:2.0:0.6 & 0.035 & $\mathrm{C} / \mathrm{C}$ & Cyto \\
\hline
\end{tabular}
kinase 
Table 1 Differentially expressed proteins identified by MALDI-TOF/TOF-MS at five grain developmental stages in bread wheat cultivars Jimai 20 and Zhoumai 16

\section{(Continued)}

\begin{tabular}{|c|c|c|}
\hline 18 & gi/18076790 & Phosphoglucomutase (PGM) \\
\hline 19 & gi/18076790 & Phosphoglucomutase (PGM) \\
\hline 20 & gi/226316441 & Fructose-bisphosphate aldola \\
\hline 21 & gi/18496065 & $\begin{array}{l}\text { Putative fructose } \\
\text { 1-,6-biphosphate aldolase }\end{array}$ \\
\hline 26 & gi/1785948 & $\begin{array}{l}\text { Cytosolic triosephosphate } \\
\text { isomerase }\end{array}$ \\
\hline 27 & gi/11124572 & Triosephosphat-isomerase \\
\hline 29 & gi/11124572 & Triosephosphat-isomerase \\
\hline 37 & gi/133872550 & Bp2A protein \\
\hline
\end{tabular}

3. Alcoholic fermentation:

\section{8 gi/32400847 Formate dehydrogenase}

39 gi/32400847 Formate dehydrogenase

\section{Starch metabolism:}

$\begin{array}{ccl}35 & \text { gi/21680 } & \begin{array}{l}\text { ADP-glucose } \\ \text { pyrophosophorylase } \\ \text { preprotein }\end{array} \\ 41 & \text { QOPG36 } & \begin{array}{l}\text { Glucose-1-phosphate } \\ \text { adenylyltransferase } \\ \text { Glucose-1-phosphate } \\ \text { adenylyltransferase } \\ \text { Glucose-1-phosphate } \\ \text { adenylyltransferase }\end{array}\end{array}$

\section{Lipid and sterol metabolism:}
30 gi/167113 Aldose reductase-related
$31 \mathrm{gi} / 167113 \quad$ Aldose reductase-related protein
40 gi/218157 Cytoplasmic aldolase
145 gi/66840998 5a2 protein

\section{Protein synthesis/assembly/degradation}
36 gi/41052632 Peptidylprolyl isomerase Cyp2
54 gi/154761388 Cyclophilin
55 gi/154761388 Cyclophilin
56 gi/154761388 Cyclophilin

\begin{tabular}{|c|c|c|c|c|c|c|c|c|c|c|}
\hline T. aestivum & 100 & 73 & 2 & $5.84 / 5.66$ & 1:1.3:2.3:0.9:0.8 & 0.017 & 1:1.7:3.9:0.6:0.4 & 0.029 & $C / C$ & Cyto \\
\hline T. aestivum & 98.67 & 47 & 1 & $5.85 / 5.66$ & 1:0.7:0.2:0:0 & 0.026 & 1:0.5:0.2:0:0 & 0.015 & $\mathrm{~A} / \mathrm{A}$ & Cyto \\
\hline T. aestivum & 100 & 64 & 2 & $6.33 / 6.85$ & 0:0:1.0:3.1:3.0 & 0.023 & 0:0:1.0:4.1:4.7 & 0.016 & $\mathrm{~B} / \mathrm{B}$ & Cyto \\
\hline T. aestivum & 98.12 & 84 & 1 & $6.15 / 8.71$ & $1: 0.5: 0.2: 0: 0$ & 0.034 & 1:0.4:0.2:0:0 & 0.034 & A/A & Cyto \\
\hline H. vulgare & 100 & 148 & 2 & $5.18 / 5.39$ & 1:1.1:0.7:0.5:1.4 & 0.036 & 1:2.1:1.6:2.0:1.7 & 0.025 & $E / E$ & Cyto \\
\hline T. aestivum & 100 & 116 & 2 & $4.46 / 5.38$ & 1:1.4:0.6:0:0 & 0.013 & 1:0.8:0.4:0:0 & 0.022 & C/A & Cyto \\
\hline T. aestivum & 100 & 235 & 3 & $5.72 / 5.38$ & 1:1.4:0.8:0.5:0.2 & 0.012 & 1:0.9:0.5:0.3:0 & 0.037 & $C / A$ & Cyto \\
\hline T. aestivum & 100 & 132 & 3 & $5.38 / 5.86$ & 1:1.5:1:0.7:0.2 & 0.022 & 1:0.8:0.6:0.5:0 & 0.033 & C/A & Cyto \\
\hline T. aestivum & 100 & 68 & 2 & $6.02 / 8.61$ & 0:0:1.0:2.6:2.9 & 0.033 & 0:0:0:1.0:1.5 & 0.019 & $\mathrm{~B} / \mathrm{B}$ & Cyto \\
\hline T. aestivum & 100 & 112 & 3 & $6.45 / 8.61$ & 0:0:1.0:3.4:6.1 & 0.031 & 0:0:1.0:3.2:7.8 & 0.028 & $\mathrm{~B} / \mathrm{B}$ & Cyto \\
\hline T. aestivum & 95.78 & 54 & 2 & $6.35 / 6.61$ & 1:0.8:0.7:0.6:0.4 & 0.027 & 1:0.8:0.6:0.6:0.3 & 0.027 & $\mathrm{~A} / \mathrm{A}$ & $P$ \\
\hline T. aestivum & 100 & 183 & 5 & $6.02 / 6.12$ & 1:1.3:0.4:0.2:0.2 & 0.029 & 1:1.2:0.4:0.2:0.1 & 0.034 & $C / C$ & $P$ \\
\hline T. aestivum & 100 & 110 & 3 & $6.42 / 6.12$ & 1:0.9:0.6:0.4:0.2 & 0.021 & 1:1.5:0.8:0.4:0.3 & 0.024 & $\mathrm{~A} / \mathrm{C}$ & $P$ \\
\hline T. aestivum & 100 & 125 & 3 & $6.23 / 5.89$ & 1:0.8:0.5:0.2:0.1 & 0.037 & 1:1.3:0.6:0.3:0.2 & 0.016 & $\mathrm{~A} / \mathrm{C}$ & $P$ \\
\hline B. inermis & 98.12 & 101 & 1 & $6.19 / 6.28$ & 0:0:0:1.0:1.8 & 0.034 & 0:0:0:1.0:6.8 & 0.025 & $\mathrm{~B} / \mathrm{B}$ & Cyto \\
\hline B. inermis & 99.83 & 116 & 2 & $6.17 / 6.28$ & 0:0:0:1.0:0.8 & 0.036 & 0:0:1.0:1.8:1.5 & 0.028 & $C / C$ & Cyto \\
\hline D. sativajaponica & 100 & 110 & 2 & $6.65 / 6.56$ & 1:0.6:0.3:0.2:0.1 & 0.031 & 1:0.9:0.4:0.3:0.1 & 0.019 & $\mathrm{~A} / \mathrm{A}$ & Cyto \\
\hline T. aestivum & 100 & 187 & 4 & $5.84 / 8.38$ & $1: 8.3: 3.5: 2.1: 2.4$ & 0.038 & 1:1.1:0.5:0.7:0.7 & 0.024 & $C / C$ & $P$ \\
\hline D. sativajaponica & 96.38 & 50 & 2 & 8.97/8.61 & 1:0.2:0.8:0.9:1.1 & 0.012 & 1:0.5:0.5:0.4:0.8 & 0.014 & $\mathrm{D} / \mathrm{D}$ & Cyto \\
\hline T. aestivum & 98.21 & 19 & 2 & $8.39 / 8.59$ & 1:0.3:1.3:1.4:1.6 & 0.027 & 1:0.5:0.4:0.4:0.8 & 0.011 & $\mathrm{D} / \mathrm{D}$ & Cyto \\
\hline T. aestivum & 100 & 84 & 3 & $9.12 / 8.59$ & 1:0.2:0.8:0.9:1.1 & 0.031 & 1:0.6:0.5:0.5:0.9 & 0.009 & $\mathrm{D} / \mathrm{D}$ & Cyto \\
\hline T. aestivum & 100 & 95 & 3 & $6.28 / 8.59$ & $0: 0: 0: 1.0: 6.8$ & 0.013 & - & - & $B /-$ & Cyto \\
\hline
\end{tabular}


Table 1 Differentially expressed proteins identified by MALDI-TOF/TOF-MS at five grain developmental stages in bread wheat cultivars Jimai 20 and Zhoumai 16

(Continued)

\begin{tabular}{|c|c|c|c|c|c|c|c|c|c|c|c|c|c|}
\hline 59 & gi/32352154 & $\begin{array}{l}\text { Nascent polypeptide associated } \\
\text { complex alpha chain }\end{array}$ & O. sativajaponica & 100 & 83 & 1 & $4.17 / 4.34$ & $0: 0: 0: 1.0: 2.1$ & 0.016 & 0:0:0:1.0:3.0 & 0.028 & $\mathrm{~B} / \mathrm{B}$ & Cyto \\
\hline 62 & gi/2492077 & $\begin{array}{l}\text { Sequence } 5 \text { from patent } \\
\text { US } 5668007\end{array}$ & T. aestivum & 100 & 189 & 2 & $6.68 / 6.77$ & $0: 1.0: 3.5: 2.1: 5.3$ & 0.027 & 0:1.0:0.9:3.0:6.7 & 0.017 & $\mathrm{~B} / \mathrm{B}$ & Cyto \\
\hline 106 & gi/75279909 & $\begin{array}{l}\text { Serpin-Z2B; AltName: } \\
\text { Full = TriaeZ2b; }\end{array}$ & T. aestivum & 100 & 213 & 4 & $5.24 / 5.18$ & 0:0:0:1.0:0.6 & 0.021 & 0:0:1.0:1.7:0.5 & 0.016 & $\mathrm{C} / \mathrm{C}$ & $P$ \\
\hline 107 & gi/75279909 & $\begin{array}{l}\text { Serpin-Z2B; AltName: } \\
\text { Full = TriaeZ2b; }\end{array}$ & T. aestivum & 100 & 256 & 4 & $5.32 / 5.18$ & 1:1.8:2.4:1.4:0.9 & 0.023 & 0:0:1.0:1.7:1.4 & 0.021 & $\mathrm{C} / \mathrm{C}$ & P \\
\hline 128 & gi/12229936 & $\begin{array}{l}\text { Proteasome subunit alpha } \\
\text { type } 7 \text { ( } 20 \text { S proteasome } \\
\text { alpha subunit D) }\end{array}$ & C. arietinum & 100 & 110 & 2 & $12.10 / 6.86$ & 1:1.1:5.1:5.9:8.3 & 0.032 & 1:1.3:3.2:3.9:6.3 & 0.033 & $\mathrm{~B} / \mathrm{B}$ & $P$ \\
\hline \multicolumn{14}{|c|}{ Storage proteins } \\
\hline 67 & gi/171027826 & Triticin & T. aestivum & 100 & 84 & 2 & $8.94 / 6.43$ & 0:0:1.0:1.4:3.0 & 0.042 & 0:0:1.0:2.0:3.3 & 0.045 & $\mathrm{~B} / \mathrm{B}$ & ER \\
\hline 68 & gi/171027826 & Triticin & T. aestivum & 100 & 475 & 6 & $6.35 / 6.43$ & 0:0:1.0:2.1:5.5 & 0.039 & 0:0:1.0:2.4:6.5 & 0.041 & $\mathrm{~B} / \mathrm{B}$ & ER \\
\hline 69 & gi/7548844 & Triticin precursor & T. aestivum & 99.92 & 169 & 3 & $6.05 / 9.37$ & 1:0.8:0.5:0.6:0.4 & 0.026 & 1:0.4:0.5:0.4:0.3 & 0.038 & $\mathrm{~A} / \mathrm{A}$ & ER \\
\hline 70 & gi/215398470 & Globulin 3 & T. aestivum & 100 & 471 & 5 & $7.24 / 7.78$ & $0: 0: 0: 1.0: 2.6$ & 0.019 & $0: 0: 0: 1.0: 2.0$ & 0.008 & $\mathrm{~B} / \mathrm{B}$ & ER \\
\hline 71 & gi/215398470 & Globulin 3 & T. aestivum & 100 & 148 & 5 & 7.43/7.78 & $0: 0: 0: 1.0: 6.1$ & 0.025 & $0: 0: 0: 1.0: 3.9$ & 0.010 & $\mathrm{~B} / \mathrm{B}$ & ER \\
\hline 72 & gi/89143122 & $\begin{array}{l}\text { Putative avenin-like } \\
\text { b precursor }\end{array}$ & T. aestivum & 100 & 166 & 3 & $6.88 / 8.08$ & 0:0:1.0:3.3:2.1 & 0.010 & 1.0:1.7:6.5:2.9:5. & 0.034 & $\mathrm{C} / \mathrm{C}$ & ER \\
\hline 73 & gi/145321072 & Avenin-like protein & T. aestivum & 100 & 97 & 2 & $6.63 / 8.29$ & 0:0:1.0:3.2:4.1 & 0.024 & 0:0:1.0:3.5:4.0 & 0.031 & $\mathrm{~B} / \mathrm{B}$ & ER \\
\hline 74 & gi/209971847 & Gamma-gliadin & T. aestivum & 99.92 & 70 & 1 & $6.84 / 7.55$ & 0:0:1.0:2.0:4.8 & 0.029 & 1.0:1.7:6.5:9.4:12.8 & 0.028 & $\mathrm{~B} / \mathrm{B}$ & ER \\
\hline 75 & gi/133741924 & Gamma gliadin & T. aestivum & 100 & 93 & 2 & $5.33 / 8.88$ & 1:2.5:4.2:9.9:11.9 & 0.020 & 1:2.2:3.9:5.9:6.4 & 0.022 & $\mathrm{~B} / \mathrm{B}$ & ER \\
\hline 76 & gi/133741924 & Gamma gliadin & T. aestivum & 98.12 & 52 & 2 & $5.56 / 8.88$ & 0:1:2.9:3.8:4.7 & 0.034 & 0:0:1.0:1.4:2.2 & 0.036 & $\mathrm{~B} / \mathrm{B}$ & ER \\
\hline 77 & gi/209971907 & Gamma-gliadin & T. aestivum & 97.35 & 55 & 2 & $8.55 / 8.64$ & $0: 1: 2.0: 2.2: 4.2$ & 0.031 & 0:0:1.0:1.6:2.1 & 0.031 & $\mathrm{~B} / \mathrm{B}$ & ER \\
\hline 78 & gi/164470668 & $\begin{array}{l}\text { LMW-s glutenin subunit } \\
\text { 0359D24-S }\end{array}$ & T. aestivum & 99.83 & 60 & 2 & $8.40 / 8.48$ & 0:0:1:3.6:5.0 & 0.027 & $0: 0: 0: 1.0: 1.2$ & 0.030 & $B / B$ & ER \\
\hline 79 & gi/47607146 & $\begin{array}{l}\text { S-type low molecular } \\
\text { weight glutenin } L 4-55\end{array}$ & T. aestivum & 100 & 90 & 2 & $8.67 / 8.51$ & $0: 0: 0: 1.0: 2.9$ & 0.024 & - & - & $B /-$ & Cyto \\
\hline 80 & gi/215398468 & Globulin 3C & T. aestivum & 100 & 80 & 2 & $6.37 / 9.15$ & 0:0:0:1.0:1.6 & 0.026 & $0: 0: 0: 1.0: 2.0$ & 0.041 & $\mathrm{~B} / \mathrm{B}$ & ER \\
\hline 81 & gi/421978 & Globulin Beg1 precursor & H. vulgare & 100 & 85 & 2 & $5.64 / 6.81$ & $0: 0: 0: 1.0: 4.2$ & 0.031 & 0:0:0:1.0:3.0 & 0.044 & $\mathrm{~B} / \mathrm{B}$ & ER \\
\hline 82 & gi/167004 & Embryo globulin & $\begin{array}{l}\text { H. vulgare } \\
\text { subsp. vulgare }\end{array}$ & 95.67 & 53 & 1 & $6.79 / 6.81$ & $0: 0: 0: 1.0: 4.4$ & 0.011 & $0: 0: 0: 1.0: 2.5$ & 0.025 & $\mathrm{~B} / \mathrm{B}$ & ER \\
\hline 84 & gi/170696 & Storage protein & T. aestivum & 100 & 52 & 1 & $9.49 / 6.82$ & 0:0:1.0:1.4:1.7 & 0.012 & 0:0:1.0:0.8:2.0 & 0.018 & $\mathrm{~B} / \mathrm{B}$ & ER \\
\hline \multicolumn{14}{|c|}{ Nitrogen metabolism } \\
\hline 47 & gi/164471780 & Aspartate aminotransferase & T. aestivum & 99.96 & 25 & 2 & $6.61 / 6.77$ & 1:1.3:1.0:0.6:0.1 & 0.019 & 1:0.9:0.8:0.6:0.2 & 0.027 & $C / A$ & Cyto \\
\hline 48 & gi/164471780 & Aspartate aminotransferase & T. aestivum & 100 & 25 & 2 & $6.95 / 6.77$ & $1: 0.8: 0.5: 0.2: 0.3$ & 0.021 & $1: 0.6: 0.5: 0.2: 0.2$ & 0.024 & A/A & Cyto \\
\hline
\end{tabular}


Table 1 Differentially expressed proteins identified by MALDI-TOF/TOF-MS at five grain developmental stages in bread wheat cultivars Jimai 20 and Zhoumai 16 (Continued)

\begin{tabular}{|c|c|c|c|c|c|c|c|c|c|c|c|c|c|}
\hline 49 & gi/584706 & $\begin{array}{l}\text { Aspartate aminotransferase, } \\
\text { cytoplasmic }\end{array}$ & O. sativajaponica & 100 & 159 & 3 & $6.89 / 7.75$ & - & - & 1:0.7:0.5:0:0 & 0.031 & $-/ A$ & Cyto \\
\hline 51 & gi/14018051 & $\begin{array}{l}\text { Putative alanine } \\
\text { aminotransferase }\end{array}$ & O. sativajaponica & 100 & 107 & 3 & $6.11 / 6.23$ & $1: 2.4: 3.3: 2.6: 1.5$ & 0.034 & 1:3.4:2.1:2.1:1.1 & 0.034 & $\mathrm{C} / \mathrm{C}$ & Cyto \\
\hline 52 & gi/56315117 & $\begin{array}{l}\text { Predicted serine-pyruvate } \\
\text { aminotransferase }\end{array}$ & O. sativajaponica & 100 & 113 & 1 & $6.18 / 5.86$ & $0: 0: 0: 1.0: 2.7$ & 0.031 & $0: 0: 0: 1.0: 1.7$ & 0.018 & $\mathrm{~B} / \mathrm{B}$ & Cyto \\
\hline 53 & gi/6006863 & $\begin{array}{l}\text { Putative methionine } \\
\text { synthase }\end{array}$ & A. thaliana & 98.76 & 69 & 1 & $5.88 / 6.09$ & 1:2.4:3.3:2.6:1.5 & 0.030 & $1: 0.7: 0.5: 0: 0$ & 0.016 & $\mathrm{C} / \mathrm{A}$ & Cyto \\
\hline \multicolumn{14}{|c|}{ Transcription/translation } \\
\hline 60 & gi/479830 & $\begin{array}{l}\text { Translation elongation } \\
\text { factor eEF-1 beta' chain }\end{array}$ & T. aestivum & 100 & 64 & 1 & $4.63 / 4.54$ & $0: 0: 0: 1.0: 2.2$ & 0.016 & $0: 0: 0: 1.0: 1.7$ & 0.028 & $\mathrm{~B} / \mathrm{B}$ & Cyto \\
\hline 85 & gi/18419557 & Transposase & T. aestivum & 97.54 & 98 & 4 & $6.53 / 9.48$ & 1:1.8:6.1:1.6:0.2 & 0.015 & 1:1.5:2.2:1.7:1.1 & 0.027 & $\mathrm{C} / \mathrm{C}$ & Nucl \\
\hline 86 & gi/30793446 & $27 \mathrm{~K}$ protein & T. aestivum & 98.12 & 71 & 1 & $6.01 / 6.06$ & - & - & 1:1.9:2.6:2.7:3.3 & 0.035 & $-/ B$ & Cyto \\
\hline 87 & gi/30793446 & $27 \mathrm{~K}$ protein & T. aestivum & 95.89 & 69 & 1 & $5.98 / 6.06$ & $0: 0: 0: 1.0: 2.1$ & 0.045 & $0: 0: 0: 1.0: 2.3$ & 0.031 & $\mathrm{~B} / \mathrm{B}$ & Cyto \\
\hline 88 & gi/30793446 & $27 \mathrm{~K}$ protein & T. aestivum & 100 & 171 & 4 & $5.64 / 6.06$ & 1:1.9:2.0:3.6:3.4 & 0.025 & 1:1.1:2.1:1.6:2.0 & 0.012 & $\mathrm{~B} / \mathrm{E}$ & Cyto \\
\hline 89 & gi/46394372 & $\begin{array}{l}\text { TPA: TPA_inf: WRKY } \\
\text { transcription factor } 59\end{array}$ & O. sativa & 95.45 & 113 & 2 & $5.46 / 7.7$ & 1:1.4:2.2:3.0:2.9 & 0.018 & 1:1.8:2.2:4.4:2.7 & 0.028 & $B / C$ & $P$ \\
\hline 90 & gi/30793446 & $27 \mathrm{~K}$ protein & T. aestivum & 100 & 60 & 1 & $6.06 / 6.06$ & $0: 0: 0: 1.0: 2.3$ & 0.021 & $0: 0: 0: 1.0: 2.2$ & 0.015 & $\mathrm{~B} / \mathrm{B}$ & Cyto \\
\hline 91 & gi/27735373 & $\begin{array}{l}\text { Replication factor } C \\
\text { like protein }\end{array}$ & T. aestivum & 100 & 64 & 1 & $5.84 / 8.92$ & 1:0.5:0.3:0.2:0.2 & 0.026 & 1:1.4:0.8:0.7:0.5 & 0.024 & $\mathrm{~A} / \mathrm{C}$ & Nucl \\
\hline 93 & Q03033 & $\begin{array}{l}\text { Elongation factor } \\
1 \text {-alpha }\end{array}$ & T. aestivum & 100 & 97 & 2 & $9.35 / 9.20$ & 0:0:0:1.0:2.1 & 0.034 & 0:0:0:1.0:2.6 & 0.026 & $\mathrm{~B} / \mathrm{B}$ & Nucl \\
\hline \multicolumn{14}{|c|}{ Signal transduction } \\
\hline 64 & gi/134290443 & $\begin{array}{l}\text { Pm3b-like disease } r \\
\text { esistance protein 15Q1 }\end{array}$ & T. aestivum & 96.58 & 96 & 2 & $7.21 / 6.27$ & 1:1.3:0.40.4:0.3 & 0.029 & 1:1.0:0.4:0.3:0.4 & 0.024 & C/A & Cyto \\
\hline 65 & gi/18145 & $\begin{array}{l}\text { Putative protein has homology } \\
\text { to } \mathrm{G} \text { proteinbeta subunit }\end{array}$ & C. reinhardtii & 100 & 60 & 1 & $5.89 / 7.59$ & 0:1.0:3.2:1.6:0 & 0.042 & 1:1.0:1.6:1.2:0 & 0.026 & $\mathrm{C} / \mathrm{C}$ & Cyto \\
\hline 66 & gi/50932677 & $\begin{array}{l}\text { Putative guanine nucleotide- } \\
\text { binding proteinbeta subunit }\end{array}$ & O. sativa & 100 & 121 & 2 & $6.07 / 6.06$ & 0:1.0:2.7:1.4:0 & 0.044 & 1:1.1:3.4:2.2:0.8 & 0.034 & $\mathrm{C} / \mathrm{C}$ & Cyto \\
\hline 142 & gi/9652119 & $\begin{array}{l}\text { Nucleoside diphosphate } \\
\text { kinase }\end{array}$ & L. perenne & 99.92 & 192 & 2 & $6.36 / 6.30$ & 1:0.9:0.7:0.4:0.2 & 0.019 & 1:0.8:0.8:0.5:0.2 & 0.021 & A/A & Cyto \\
\hline \multicolumn{14}{|c|}{ Stress/defense } \\
\hline 57 & gi/640015 & CMx & T. aestivum & 100 & 102 & 2 & $5.51 / 9.23$ & 0:0:1.0:2.5:1.8 & 0.036 & 0:0:1.0:2.8:2.4 & 0.022 & $\mathrm{C} / \mathrm{C}$ & $P$ \\
\hline 58 & gi/75107149 & $\begin{array}{l}\text { RecName: Full = Chymotrypsin } \\
\text { inhibitor WCl; AltName: } \\
\text { Full=Chloroform/ } \\
\text { methanol-solubleprotein WCI }\end{array}$ & T. aestivum & 100 & 164 & 3 & $7.34 / 7.42$ & 0:0:1.0:3.3:1.9 & 0.027 & 0:0:1.0:2.7:0.9 & 0.015 & $\mathrm{C} / \mathrm{C}$ & Cyto \\
\hline 94 & gi/38098487 & Alpha amylase inhibitor protein & T. aestivum & 99.91 & 62 & 2 & $6.65 / 7.44$ & $0: 0: 1.0: 3.4: 4.0$ & 0.021 & 0:0:1.0:3.0:3.5 & 0.018 & $\mathrm{~B} / \mathrm{B}$ & P \\
\hline
\end{tabular}




\section{Table 1 Differentially expressed proteins identified by MALDI-TOF/TOF-MS at five grain developmental stages in bread wheat cultivars Jimai 20 and Zhoumai 16}

(Continued)

\begin{tabular}{|c|c|c|c|c|c|c|c|c|c|c|c|c|c|}
\hline 95 & gi/66841026 & Alpha-amylase inhibitor 0.19 & T. aestivum & 100 & 94 & 2 & $7.72 / 6.86$ & 0:0:1.0:2.0:1.2 & 0.031 & 0:0:1.0:2.7:2.0 & 0.024 & $\mathrm{C} / \mathrm{C}$ & P \\
\hline 96 & gi/66841026 & Alpha-amylase inhibitor 0.19 & T. aestivum & 100 & 493 & 5 & $6.98 / 6.86$ & 0:0:1.0:4.3:5.0 & 0.021 & $0: 1.0: 1.7: 3.9: 2.7$ & 0.038 & $B / C$ & $P$ \\
\hline 97 & gi/123956 & $\begin{array}{l}\text { Alpha-amylase/trypsin } \\
\text { inhibitor CM2; }\end{array}$ & T. aestivum & 100 & 191 & 2 & $5.71 / 6.86$ & $0: 0: 1.0: 2.2: 1.2$ & 0.018 & $0: 1.0: 1.4: 3.5: 1.1$ & 0.016 & $\mathrm{C} / \mathrm{C}$ & Extr \\
\hline 98 & $\mathrm{gi} / 221855644$ & $\begin{array}{l}\text { Alpha-amylase inhibitor } \\
\text { CM16 subunit }\end{array}$ & T. macha & 99.96 & 60 & 2 & $4.56 / 5.31$ & 0:0:1.0:4.2:1.4 & 0.021 & $0: 0: 1 \cdot 0: 2 \cdot 3: 1.7$ & 0.035 & $\mathrm{C} / \mathrm{C}$ & Extr \\
\hline 99 & gi/221855644 & $\begin{array}{l}\text { Alpha-amylase inhibitor } \\
\text { CM16 subunit }\end{array}$ & T. macha & 100 & 97 & 2 & $5.11 / 5.31$ & 0:0:1.0:3.1:1.6 & 0.024 & $0: 1.0: 2.5: 5.4: 3.2$ & 0.026 & $\mathrm{C} / \mathrm{C}$ & Extr \\
\hline 100 & $\mathrm{gi} / 225042$ & Alpha amylase inhibitor & T. aestivum & 100 & 64 & 2 & $6.65 / 6.77$ & 1:2.7:9.4:5.5:13.9 & 0.029 & 1:4.1:10.4:6.1:13.1 & 0.036 & $E / E$ & Cyto \\
\hline 101 & gi/123955 & $\begin{array}{l}\text { Alpha-amylase/trypsin i } \\
\text { nhibitor CM1; }\end{array}$ & T. aestivum & 100 & 73 & 2 & $6.81 / 7.49$ & 0:0:1.0:3.1:3.0 & 0.012 & 1:1.0:1.4:4.1:3.3 & 0.012 & $B / C$ & Extr \\
\hline 102 & gi/54778507 & $\begin{array}{l}0.19 \text { dimeric alpha-amylase } \\
\text { inhibitor }\end{array}$ & T. aestivum & 100 & 238 & 2 & $6.76 / 5.73$ & 1:1.5:2.6:2.4:2.3 & 0.025 & 1:1.6:2.1:2.7:2.4 & 0.028 & $\mathrm{C} / \mathrm{C}$ & $P$ \\
\hline 104 & gi/408873 & $\begin{array}{l}\text { Puroindoline }=\text { basic } \\
\text { cystine-rich protein }\end{array}$ & T. aestivum & 100 & 125 & 4 & $9.05 / 5.60$ & 1:1.5:2.8:2.9:2.6 & 0.016 & 1:1.2:1.7:4.0:1.4 & 0.023 & $\mathrm{C} / \mathrm{C}$ & $P$ \\
\hline 105 & gi/408873 & $\begin{array}{l}\text { Puroindoline = basic } \\
\text { cystine-rich protein }\end{array}$ & T. aestivum & 100 & 125 & 4 & $6.73 / 8.76$ & 0:0:1.0:3.0:5.2 & 0.039 & $0: 0: 1.0: 2.0: 2.5$ & 0.045 & $B / B$ & $P$ \\
\hline 110 & gi/51247633 & $\begin{array}{l}\text { Chain A, Crystal Structure Of } \\
\text { Family } 11 \text { Xylanase In Complex } \\
\text { With Inhibitor (Xip-l) }\end{array}$ & T. aestivum & 100 & 78 & 2 & $8.24 / 5.18$ & 0:0:1.0:0.9:3.1 & 0.038 & 0:0:1.0:3.2:7.2 & 0.008 & $B / B$ & $P$ \\
\hline 111 & gi/51247633 & $\begin{array}{l}\text { Chain A, Crystal Structure Of } \\
\text { Family } 11 \text { Xylanase In Complex } \\
\text { With Inhibitor (Xip-l) }\end{array}$ & T. aestivum & 100 & 163 & 3 & $8.32 / 5.18$ & 0:0:1.0:1.4:1.5 & 0.027 & 0:0:1.0:4.5:5.8 & 0.015 & $\mathrm{~B} / \mathrm{B}$ & $P$ \\
\hline 112 & gi/62465514 & Class II chitinase & T. aestivum & 100 & 95 & 2 & $8.39 / 5.56$ & 0:0:1.0:1.1:1.8 & 0.025 & 0:0:1.0:0.7:1.7 & 0.028 & $B / B$ & Extr \\
\hline 113 & gi/62465514 & Class II chitinase & T. aestivum & 100 & 54 & 1 & $8.42 / 8.27$ & 0:0:1.0:1.1:1.6 & 0.024 & 0:0:1.0:1.2:2.0 & 0.015 & $B / B$ & Extr \\
\hline 114 & gi/62465514 & Class II chitinase & T. aestivum & 100 & 138 & 2 & $8.37 / 8.27$ & 1:2.5:5.8:3.0:9.8 & 0.025 & 1:1.2:2.1:2.1:6.2 & 0.025 & $B / B$ & Extr \\
\hline 115 & gi/62465514 & Class II chitinase & T. aestivum & 100 & 69 & 3 & $8.35 / 8.27$ & 0:0:1.0:1.2:1.9 & 0.038 & 0:0:1.0:0.8:3.9 & 0.005 & $\mathrm{~B} / \mathrm{B}$ & Extr \\
\hline 116 & gi/62465514 & Class II chitinase & T. aestivum & 100 & 206 & 4 & $7.40 / 8.66$ & 0:0:1.0:1.4:1.5 & 0.027 & 0:0:1.0:1.6:2.1 & 0.034 & $\mathrm{~B} / \mathrm{B}$ & Extr \\
\hline 117 & gi/25989705 & LEA1 protein & T. aestivum & 100 & 117 & 2 & $9.21 / 8.81$ & 0:0:0:1.0:2.9 & 0.012 & $0: 0: 0: 1.0: 2.7$ & 0.021 & $\mathrm{~B} / \mathrm{B}$ & Nucl \\
\hline 119 & gi/134034615 & $\begin{array}{l}\text { Monomeric alpha-amylase } \\
\text { inhibitor }\end{array}$ & T. aestivum & 99.98 & 45 & 2 & $5.54 / 5.37$ & $0: 0: 0: 1.0: 2.0$ & 0.019 & 0:0:0:1.0:1.6 & 0.025 & $\mathrm{~B} / \mathrm{B}$ & $P$ \\
\hline 120 & gi/134034615 & $\begin{array}{l}\text { Monomeric alpha-amylase } \\
\text { inhibitor }\end{array}$ & T. aestivum & 100 & 47 & 2 & $5.65 / 5.37$ & - & - & $0: 1.0: 1.6: 2.1: 2.7$ & 0.015 & $-/ B$ & $P$ \\
\hline 121 & gi/22001285 & Peroxidase 1 & T. aestivum & 99.99 & 58 & 3 & $7.35 / 8.14$ & 1:1.3:0.4:0.4:0.3 & 0.038 & 1:1.0:0.4:0.1:0.4 & 0.045 & $\mathrm{C} / \mathrm{A}$ & Extr \\
\hline 122 & gi/22001285 & Peroxidase 1 & T. aestivum & 100 & 266 & 4 & $8.39 / 8.14$ & 0:1.0:3.0:3.9:3.9 & 0.031 & 0:0:1.0:0.6:1.3 & 0.004 & $\mathrm{~B} / \mathrm{B}$ & Extr \\
\hline 123 & gi/22001285 & Peroxidase 1 & T. aestivum & 100 & 136 & 6 & $8.42 / 8.14$ & 0:0:1.0:0.6:0.8 & 0.029 & 0:0:1.0:0.8:1.6 & 0.026 & $C / B$ & Extr \\
\hline 124 & gi/75246527 & & T. aestivum & 100 & 203 & 3 & $4.24 / 4.55$ & 1:1.5:0.7:0.3:0.2 & 0.025 & 1:2.2:1.4:1.1:0.6 & 0.049 & $\mathrm{C} / \mathrm{C}$ & Cyto \\
\hline
\end{tabular}


Table 1 Differentially expressed proteins identified by MALDI-TOF/TOF-MS at five grain developmental stages in bread wheat cultivars Jimai 20 and Zhoumai 16 (Continued)

\begin{tabular}{|c|c|c|c|c|c|c|c|c|c|c|c|c|c|}
\hline & & $\begin{array}{l}\text { Translationally-controlled } \\
\text { tumor protein }\end{array}$ & & & & & & & & & & & \\
\hline 125 & gi/20257409 & Thaumatin-like protein & T. aestivum & 100 & 255 & 6 & $7.65 / 7.85$ & $0: 0: 0: 1.0: 6.7$ & 0.024 & $0: 0: 1 \cdot 0: 2 \cdot 3: 2 \cdot 0$ & 0.030 & $\mathrm{~B} / \mathrm{B}$ & $P$ \\
\hline 126 & gi/20257409 & Thaumatin-like protein & T. aestivum & 100 & 162 & 2 & $7.80 / 7.85$ & 0:0:0:1.0:8.9 & 0.016 & 0:0:1.0:1.3:0.9 & 0.034 & $B / C$ & $\mathrm{P}$ \\
\hline 127 & gi/226897529 & Superoxide dismutase & T. aestivum & 100 & 72 & 2 & $5.97 / 5.71$ & - & - & 1.0:2.1:1.9:4.2:3.2 & 0.015 & $-/ C$ & Cyto \\
\hline \multicolumn{14}{|c|}{ Photosynthesis } \\
\hline 132 & gi/11990897 & $\begin{array}{l}\text { Ribulose-1,5-bisphosphate } \\
\text { carboxylase/oxygenase } \\
\text { small subunit }\end{array}$ & T. aestivum & 100 & 209 & 7 & $6.20 / 8.80$ & 1.0:1.2:0.4:0.4:0.3 & 0.049 & 1:1.1:0.7:0.6:0.2 & 0.034 & $\mathrm{~A} / \mathrm{C}$ & $P$ \\
\hline 140 & gi/34393258 & $\begin{array}{l}\text { Putative Oxygen-evolving } \\
\text { enhancer } \\
\text { protein 3-1,chloroplast } \\
\text { precursor }\end{array}$ & O. sativajaponica & 99.83 & 59 & 1 & $9.45 / 9.82$ & $1: 0.6: 0.5: 0.4: 0.4$ & 0.018 & 1:0.7:0.6:0.4:0.4 & 0.031 & $\mathrm{~A} / \mathrm{A}$ & $P$ \\
\hline \multicolumn{14}{|c|}{ Others } \\
\hline 143 & gi/357152329 & $\begin{array}{l}\text { Probable beta-D-xylosidase } \\
\text { 7-like }\end{array}$ & T. aestivum & 100 & 47 & 2 & $7.89 / 7.53$ & 0:0:1.0:0.5:0.5 & 0.042 & 0:0:1.0:0.5:0.5 & 0.049 & $\mathrm{C} / \mathrm{C}$ & $P$ \\
\hline 146 & gi/2454602 & Barperm1 & H. vulgare subsp. vulgare & 100 & 207 & 4 & $8.09 / 8.15$ & 0:0:1.0:1.6:0.4 & 0.018 & $0: 0: 1 \cdot 0: 1 \cdot 6: 0.5$ & 0.045 & $\mathrm{C} / \mathrm{C}$ & $P$ \\
\hline 147 & gi/115458852 & Os04g0465600 & O. sativajaponica & 100 & 92 & 1 & $7.41 / 4.75$ & 0:0:1.0:1.1:1.8 & 0.021 & 0:0:1.0:1.1:1.8 & 0.025 & $B / B$ & Cyto \\
\hline
\end{tabular}

${ }^{\mathrm{a} S p o t}$ no: corresponds to protein spot on gels shown in Figure 2 and Figure 3.

${ }^{\mathrm{b}}$ Accession no: predicted protein in NCBInr and UniprotKB database.

${ }^{c}$ Protein Score C.I.\%: the PMF score percentage of protein sequence (Confidence interval: Protein Score C.I.\% $\geq 95 \%$ ).

dMP: Matched peptides.

${ }^{e} E p l / T p l: p /$ of protein on the $\mathrm{gel} / \mathrm{p} /$ of predicted protein

fHCP(J/Z): hierachical cluster pattern A-E (Jimai 20/Zhoumai 16).

${ }^{9}$ Cyto: cytosol; P: plastid; Mito: mitochondria; ER: endoplasmic reticulum; Nucl: nuclear; Extr: extracellular. 


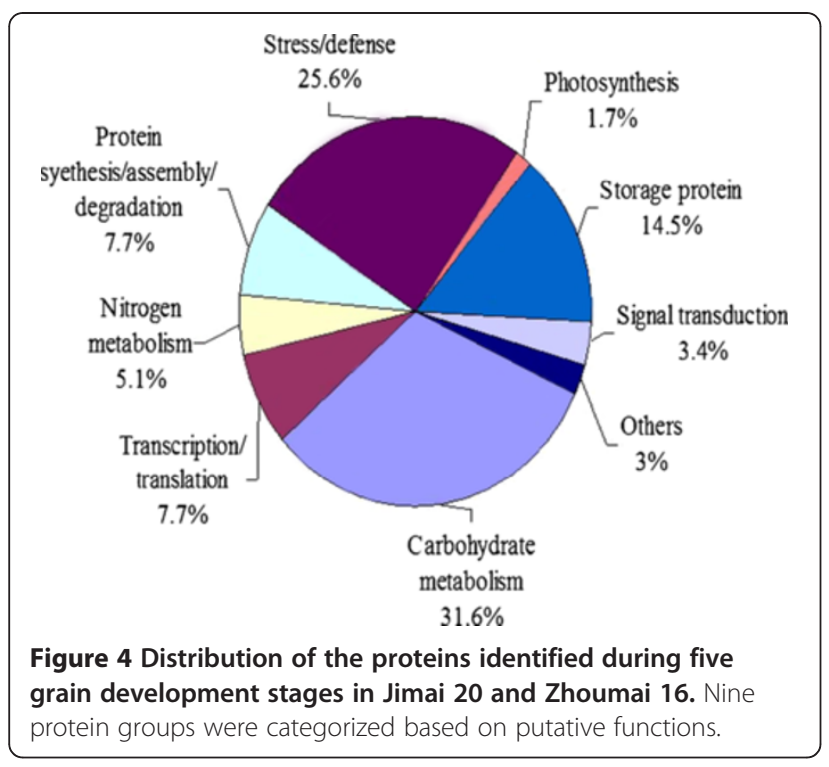

clearly reflected three distinct grain development phases: differentiation (I-II), grain filling (II-IV) and desiccation/ maturation (IV-V) as shown in Figure 6 and 7.

Expression pattern A included 19 spots in Jimai 20 and 24 in Zhoumai 16 exhibiting down-regulated modes during grain development. For example, triticin precursor (spot 69) was highly accumulated in the first two stages but was down-regulated at grain filling and maturity. Most of the proteins involved in the TCA pathway and glycolysis showed this expression pattern, such as isocitrate dehydrogenase NADP-dependent and its precursor (spots 32 and 33), and phosphoglycerate mutase (spots 1 and 2). Preprotein of glucose-1-phosphate adenylyltransferase (spot 35) also displayed this pattern in both cultivars.

Expression pattern B included the largest proportion of identified proteins and showed up-regulated expression, especially during the late grain development stages. Totals of 48 spots in Jimai 20 and 44 in Zhoumai 16 were in this expression group, which contained most of the

Table 2 Phosphorylated proteins stained by Pro-Q Diamond and identified by MALDI-TOF/TOF-MS during grain filling

\begin{tabular}{|c|c|c|c|c|c|c|c|c|c|c|c|}
\hline \multirow[t]{2}{*}{ Spot no. } & \multirow[t]{2}{*}{$\begin{array}{l}\text { Accession } \\
\text { no. }\end{array}$} & \multirow[t]{2}{*}{ Protein name } & \multirow[t]{2}{*}{$\begin{array}{c}\text { Protein score } \\
\text { C.I. } \%\end{array}$} & \multirow[t]{2}{*}{$\begin{array}{c}\text { Total } \\
\text { lon } \\
\text { score }\end{array}$} & \multirow[t]{2}{*}{$\begin{array}{l}\text { Total } \\
\text { lon } \\
\text { C.I.\% }\end{array}$} & \multirow[t]{2}{*}{$\begin{array}{l}\text { Theoretical } \\
\text { pl/MW(kDa) }\end{array}$} & \multirow[t]{2}{*}{$\begin{array}{l}\text { Experimental } \\
\text { pl/MW(kDa) }\end{array}$} & \multirow[t]{2}{*}{$\begin{array}{l}\text { Matched } \\
\text { sequences }\end{array}$} & \multicolumn{3}{|c|}{$\begin{array}{c}\text { Predicted } \\
\text { number of } \\
\text { phosphorylation }^{\text {sites }} \\
\end{array}$} \\
\hline & & & & & & & & & Ser & Thr & Tyr \\
\hline $67^{*}$ & gi/171027826 & Triticin & 100 & 84 & 100 & $6.43 / 64.90$ & $8.79 / 30.52$ & 2 & 18 & 2 & 4 \\
\hline $70^{*}$ & $\mathrm{gi} / 215398470$ & Globulin 3 & 100 & 471 & 100 & $7.78 / 66.31$ & $7.58 / 63.57$ & 6 & 17 & 6 & 2 \\
\hline $94^{*}$ & gi/38098487 & $\begin{array}{l}\text { Alpha amylase } \\
\text { inhibitor protein }\end{array}$ & 100 & 152 & 100 & $7.44 / 18.21$ & $6.10 / 16.70$ & 3 & 2 & 1 & 0 \\
\hline $96^{*}$ & gi/54778501 & $\begin{array}{l}0.19 \text { dimeric alpha-amylase } \\
\text { inhibitor }\end{array}$ & 100 & 360 & 100 & $6.66 / 13.33$ & $6.29 / 16.79$ & 4 & & & \\
\hline $100^{*}$ & gi/225042 & Alpha amylase inhibitor & 100 & 259 & 100 & $6.77 / 19.62$ & $6.87 / 20.32$ & 4 & 8 & 2 & 3 \\
\hline $102^{*}$ & gi/54778507 & $\begin{array}{l}0.19 \text { dimeric alpha-amylase } \\
\text { inhibitor }\end{array}$ & 100 & 373 & 100 & $5.73 / 13.24$ & $6.07 / 15.90$ & 4 & 2 & 0 & 2 \\
\hline $112^{*}$ & gi/62465514 & Class II chitinase & 100 & 95 & 100 & $8.66 / 28.20$ & $8.81 / 30.60$ & 2 & 9 & 3 & 1 \\
\hline $113^{*}$ & gi/62465514 & Class II chitinase & 100 & 69 & 99.38 & $8.66 / 28.20$ & $9.23 / 30.23$ & 1 & 9 & 3 & 1 \\
\hline $114^{*}$ & gi/62465514 & Class II chitinase & 100 & 138 & 100 & $8.66 / 28.20$ & $8.52 / 30.56$ & 2 & 9 & 3 & 1 \\
\hline $122^{*}$ & gi/22001285 & Peroxidase 1 & 100 & 138 & 100 & $8.14 / 38.80$ & $8.55 / 51.53$ & 4 & 11 & 5 & 2 \\
\hline $123^{*}$ & gi/22001285 & Peroxidase 1 & 100 & 206 & 100 & $8.14 / 38.80$ & $8.22 / 51.66$ & 4 & 11 & 5 & 2 \\
\hline$a^{\#}$ & gi/4558484 & Heat shock protein 101 & 100 & 288 & 100 & $5.95 / 101.06$ & $5.20 / 90.65$ & 6 & 21 & 3 & 7 \\
\hline$b^{\#}$ & $\mathrm{gi} / 4204859$ & Heat shock protein 80 & 100 & 286 & 100 & $5.00 / 80.33$ & $3.29 / 83.11$ & 5 & 24 & 9 & 6 \\
\hline$c^{\#}$ & $\mathrm{gi} / 18146829$ & Chitinase 3 & 100 & 63 & 100 & $6.89 / 33.46$ & $8.42 / 31.12$ & 1 & 10 & 6 & 4 \\
\hline$d^{\#}$ & gi/34925030 & $\begin{array}{l}\text { RecName: Full = Wheatwin-1; } \\
\text { AltName:Full = } \\
\text { Pathogenesis-related } \\
\text { protein } 4 a\end{array}$ & 100 & 275 & 100 & $7.57 / 15.62$ & $5.97 / 15.54$ & 4 & 2 & 2 & 1 \\
\hline$e^{\#}$ & gi/34925032 & $\begin{array}{l}\text { RecName: Full = Wheatwin-2; } \\
\text { AltName:Full = } \\
\text { Pathogenesis-related } \\
\text { protein } 4 \mathrm{~b}\end{array}$ & 100 & 224 & 100 & $8.18 / 15.86$ & $6.88 / 15.63$ & 4 & 2 & 2 & 1 \\
\hline
\end{tabular}

aNumber of phosphorylation sites predicted by NetPhos 2.0 Server.

* Spot numbers correspond to those shown in Figure 2 and Figure 3.

"Newly identified phosphorylated protein spots. 


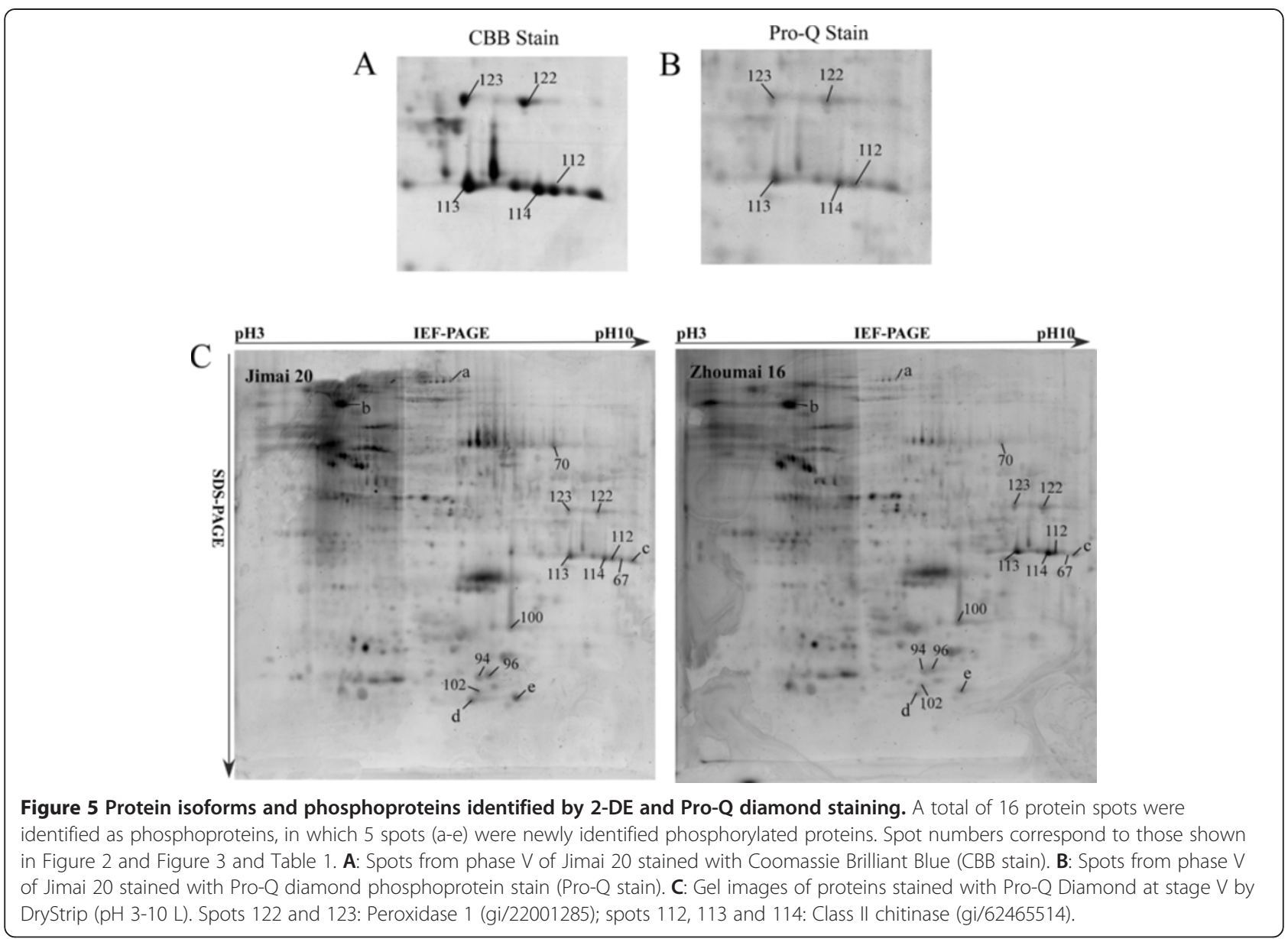

storage proteins, many stress/defense-related proteins and two enzymes involved in alcoholic fermentation. In general, storage proteins including globulins (spots 70, 71, 80 and 82), gliadins (spots 74, 75, 76 and 77) and glutenins (spot 78), triticins (spots 67 and 68) and avenin-like protein (spot 73) accumulated significantly at the later developmental stages, but only had trace expression levels during the earlier developmental stages in both cultivars. The same responses also occurred for formate dehydrogenase (spot 38 and 39) involved in alcoholic fermentation, and class II chitinase (spots 112-116) involved in stress/defense pathways. Three spots (121, 122 and 123) were identified as peroxidase 1, of which 122 displayed B expression pattern in both cultivars.

Expression pattern $\mathrm{C}$ was the second largest group of identified proteins, represented by 41 spots in Jimai 20 and 40 in Zhoumai 16, many of which included glycolysis and stress/defence-related proteins and showed upregulated expression at the early development stages, and were then down-regulated with advancement of grain filling and maturity. Representative proteins involved in this expression group were phosphoglucomutase (spot 18) and glucose-1-phosphate adenylyltransferase (spot 41) related to starch synthesis.

Expression pattern D, unlike pattern C, displayed a down to up-regulated expression trend. Only spots 36, 54 and 55 representing two proteins (peptidylprolyl isomerase Cyp2 and cyclophiliwere) showed this pattern. The remaining two spots (26 and 100) in Jimai 20 and three $(26,88$ and 100) in Zhoumai 16 accumulated a complicated pattern $\mathrm{E}$ that fluctuated during gain development (Figure 6 and 7).

\section{Comparative proteome characterization in Jimai 20 and Zhoumai 16 during grain development}

Comparative proteomic analysis demonstrated a considerable proteome expression difference between Jimai 20 and Zhoumai 16 during grain development. A total of 20 protein spots co-accumulated in both wheat cultivars, but with different expression patterns (Figure 6). For example, starch-rating enzyme glucose-1-phosphate adenyl-transferase (AGPase, spots 44 and 45) displayed A expression patterns in Jimai 20, but $\mathrm{C}$ patterns in 
Figure 6 Hirerarchical clustering analysis of differentially accumulated protein spots in Jimai 20 (left) and Zhoumai 16 (right). Red color: the higher abundance of protein spots; blue color: the lower abundance of protein spots.

Zhoumai 16. Spots 121 and 123 were identified as peroxidase I that showed expression pattern $\mathrm{C}$ in Jimai 20 , and patterns $\mathrm{A}$ and $\mathrm{B}$ in Zhoumai 16. In addition, $27 \mathrm{~K}$ protein (spot 86) and superoxide dismutase (spot 127) gradually accumulated in Zhoumai 16, but showed little change in Jimai 20 during all five stages.

Protein spots with two fold changes, or greater, in abundance at particular times between the two cultivars were considered to be cultivar-different proteins. Twenty seven spots showed cultivar-different during the five development stages and they were mainly involved in carbohydrate metabolism, stress/defense, protein storage and transcription/translation. Among them, spots 8, 56 and 79 (glyceraldehyde-3-phosphate dehydrogenase, cyclophilin and S-type low molecular weight glutenin L4-55) only accumulated in Jimai 20, but were not detected in Zhoumai 16 when subjected to ImageMaster ${ }^{\mathrm{TM}}$ 2D Platinum Software analysis. Another two spots, 49 and 120 (aspartate aminotransferase and monomeric alpha-amylase inhibitor), only accumulated in Zhoumai 16. In the remaining protein spots, 14 accumulated in higher abundance in Jimai 20 than in Zhoumai 16, whereas 8 were more abundantly accumulated in Zhoumai 16. For example, important proteins displaying higher levels of expression in Jimai 20 than in Zhoumai 16 included isocitrate dehydrogenase NADP-dependent (spot 33), triticin precursor (spot 69), LMW-s glutenin subunit (spot 78), and replication factor $\mathrm{C}$ like protein (spot 91) as shown in Figure 8. Some proteins displayed higher expression abundances in Zhoumai 16 than in Jimai 20, for example, phosphoglucomutase (spots 18 and 19).

\section{Transcriptional expression analysis by qRT-PCR}

Since wheat grains in stage $\mathrm{V}$ were approaching maturity and the reference gene accumulated unstably, the transcriptional expression profiles of ten representative genes from stages I to IV were investigated by qRTPCR with specific primers (Additional file 3: Table S3). As shown in Figure 9, the transcriptional expression patterns of only four protein genes (phosphoglucomutase, thaumatin-like protein, superoxide dismutase and monomeric alpha-amylase inhibitor) in both cultivars and two genes (glucose-1-phosphate adenylyltransferase and alpha amylase inhibitor protein) in Zhoumai 16 were consistent with their protein expression models. The remaining protein genes showed poor consistency between their transcriptional and translational levels. Interestingly, alpha-amylase inhibitor was shown to be 

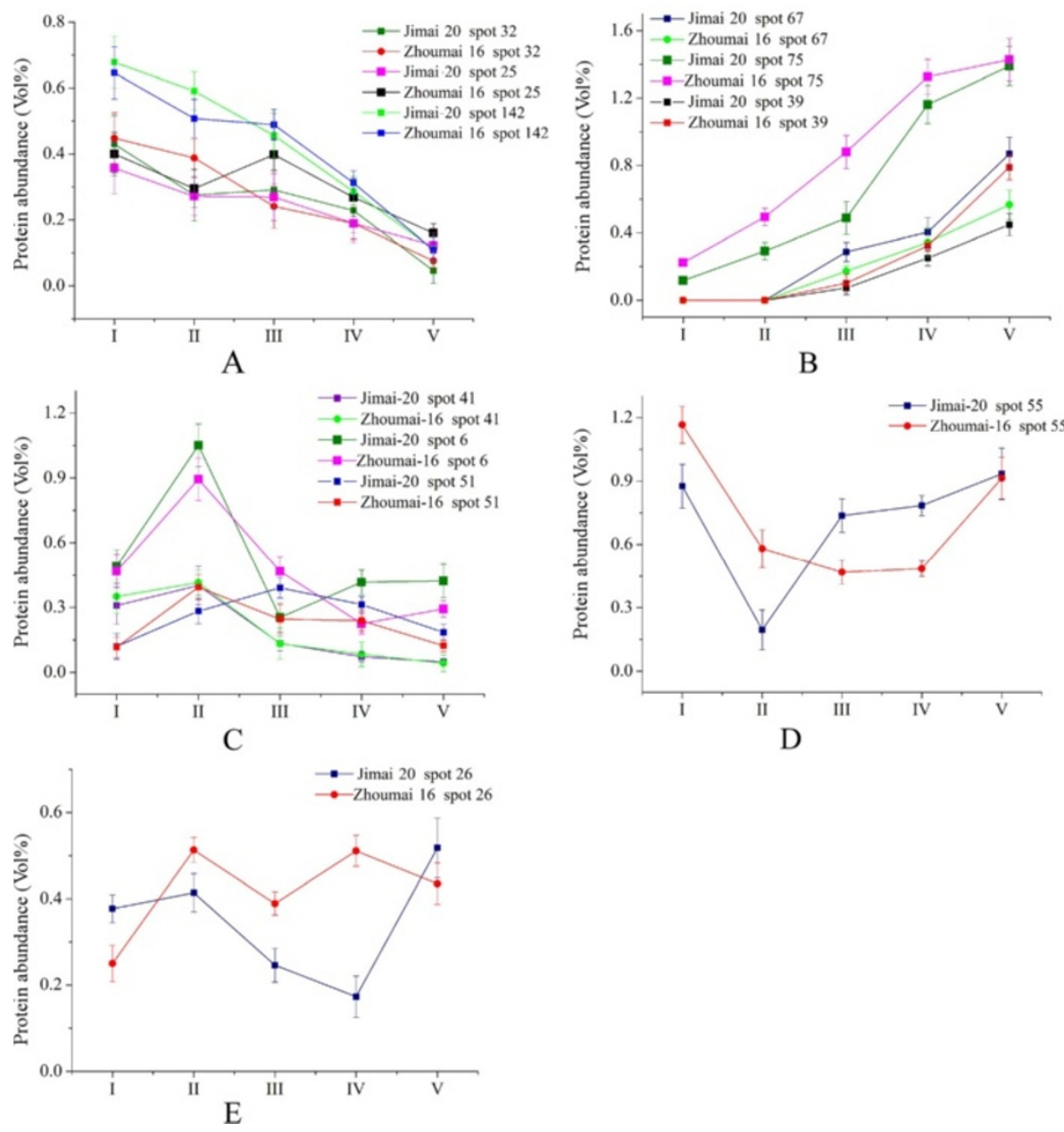

Figure 7 Protein expression patterns in Jimai 20 and Zhoumai 16 during five grain developmental stages (A-E).

phosphorylated when stained by Pro-Q Diamond dye (Table 2), a feature that might be responsible for lower consistency between transcriptional and translational expression patterns.

\section{Discussion}

In this study samples were collected during grain development and categorized according to cumulative average daily temperatures to avoid the effects of temperature on plant growth and development [37]. Proteome expression profiles during five grain development stages in two bread wheat cultivars were investigated by $2-\mathrm{DE}$ and MALDI-TOF/TOF-MS. The results revealed key molecular characteristics of grain development and provided important insights on crucial proteins and their expression profiles during specific developmental phases.

\section{Metabolism and energy supply}

The respiratory pathways of glycolysis, the tricarboxylic acid (TCA) cycle and the mitochondrial electron transport chain are essential for energy provision in a wide range of cellular functions [38]. We identified 6 spots representing 4 unique proteins related to the TCA cycle, including NAD-dependent isocitrate dehydrogenase (NAD-IDH) and NAD-IDH precursor (spots 33 and 32). NAD-IDH, present in mitochondria, is a key enzyme in the TCA cycle and catalyzes the oxidative decarboxylation of isocitrate towards 2-oxoglutarate, $\mathrm{NADH}$, and $\mathrm{CO}_{2}$. The TCA cycle-related proteins all showed high expression levels from 6 to 15 DAP (147$353^{\circ} \mathrm{Cd}$ ), and then underwent steep decreases. This implies that the TCA cycle is very active during the early grain development stages, mainly providing energy for grain development. NAD-IDH expression was higher in Jimai 20 than in Zhoumai 16 from 6 to 11 DAP, especially at 11 DPA. However, some other energy-related enzymes were accumulated as cultivar-different spots, such as phosphoglucomutase (PGM, spots 18 and 19), which were highly accumulated in Zhoumai 16 . These differential expressions in energy metabolism in different 

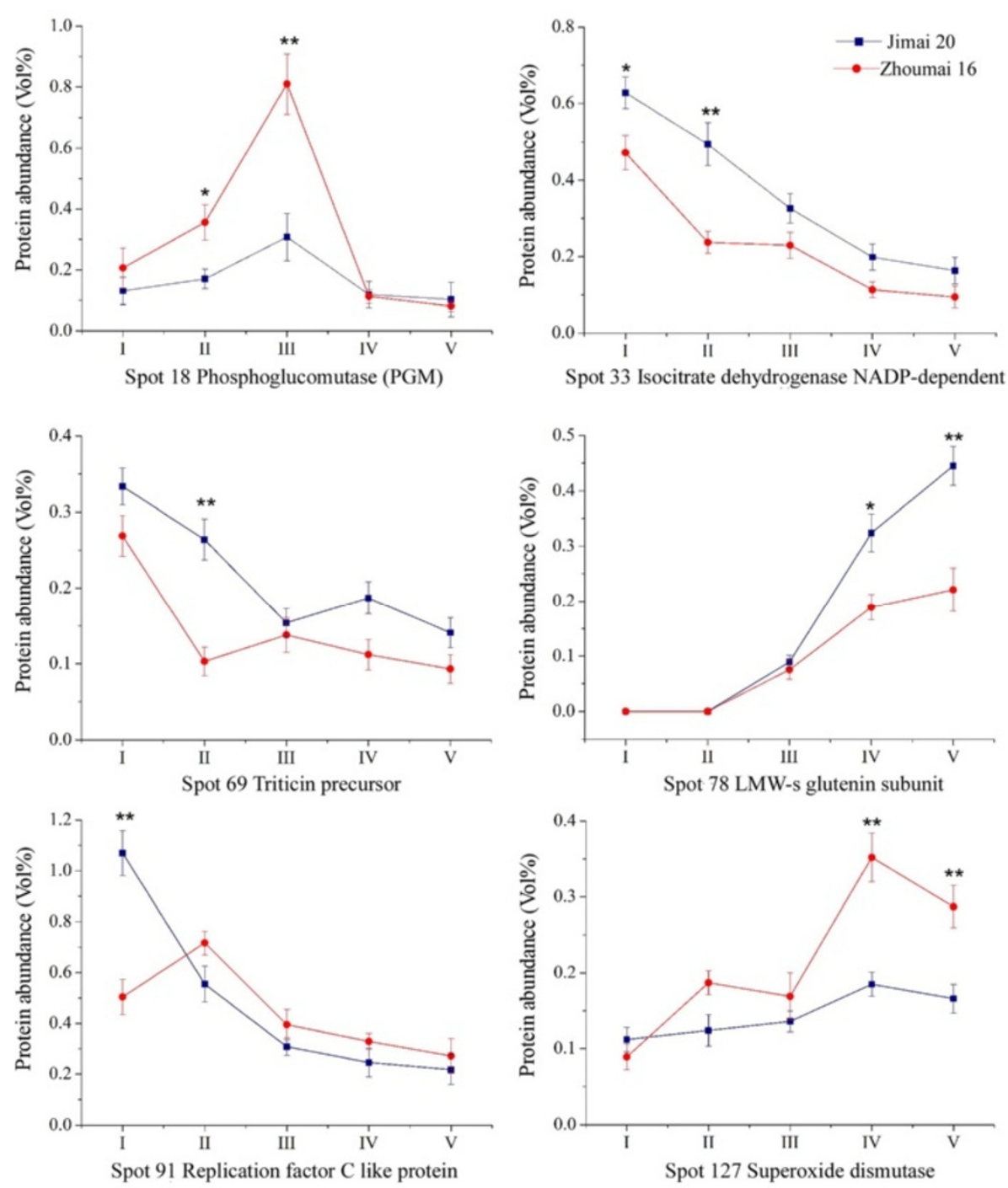

Figure 8 Differential expression of six protein spots in Jimai 20 and Zhoumai 16 during five grain development stages. Spot 18 (II/III); spot 33 (II); spot 69 (II); spot 78 (V); spot 91 (I); spot 127 (IV).

genotypes were possibly related to different cultivar performances.

For glycolysis 21 spots representing 14 unique proteins were identified. Except for a few spots such as PGM (spots 18 and 19), the vast majority of glycolysis-related proteins showed simultaneous peaks in expression around 11 DPA $\left(252^{\circ} \mathrm{Cd}\right)$ in both cultivars. This stage is consistent with cell differentiation and the beginning of dry matter accumulation during which lots of energy is needed [14]. At the same time, B-type starch granules began to form and enlarge (Figure $1 \mathrm{C}$ ), resulting in decreased $\mathrm{O}_{2}$ availability and increased energy need. Therefore, glycolysis becomes a highly significant source of energy for grain filling, which involves packaging of proteins as well as starch [16]. PGM was accumulated at higher levels during early grain development, and showed a peak at $15 \mathrm{DAP}\left(353^{\circ} \mathrm{Cd}\right)$, before decreasing to a lower constant level. This coincided with an intensive starch synthesis stage [14]. Previous reports demonstrated that PGM, as a phosphorylated protein, catalyses the bidirectional interconversion of glucose 1-phosphate (G1P) and glucose 6-phosphate (G6P) and cytosolic PGM isoforms may play an important role in the partitioning of carbon between the starch synthetic (G1Putilizing) and glycolytic (G6P-utilizing) pathways in plant tissues $[39,40]$. Phosphorylation of starch-related enzymes in wheat and maize, such as GBSS, BEIIb, SSIIa and starch phosphorylase appeared to play important roles in synthesis and accumulation, and protein phosphorylation in wheat amyloplasts could regulate starch branching enzyme activity and protein-protein interactions $[28,41,42]$. Our results also showed that PGM was 


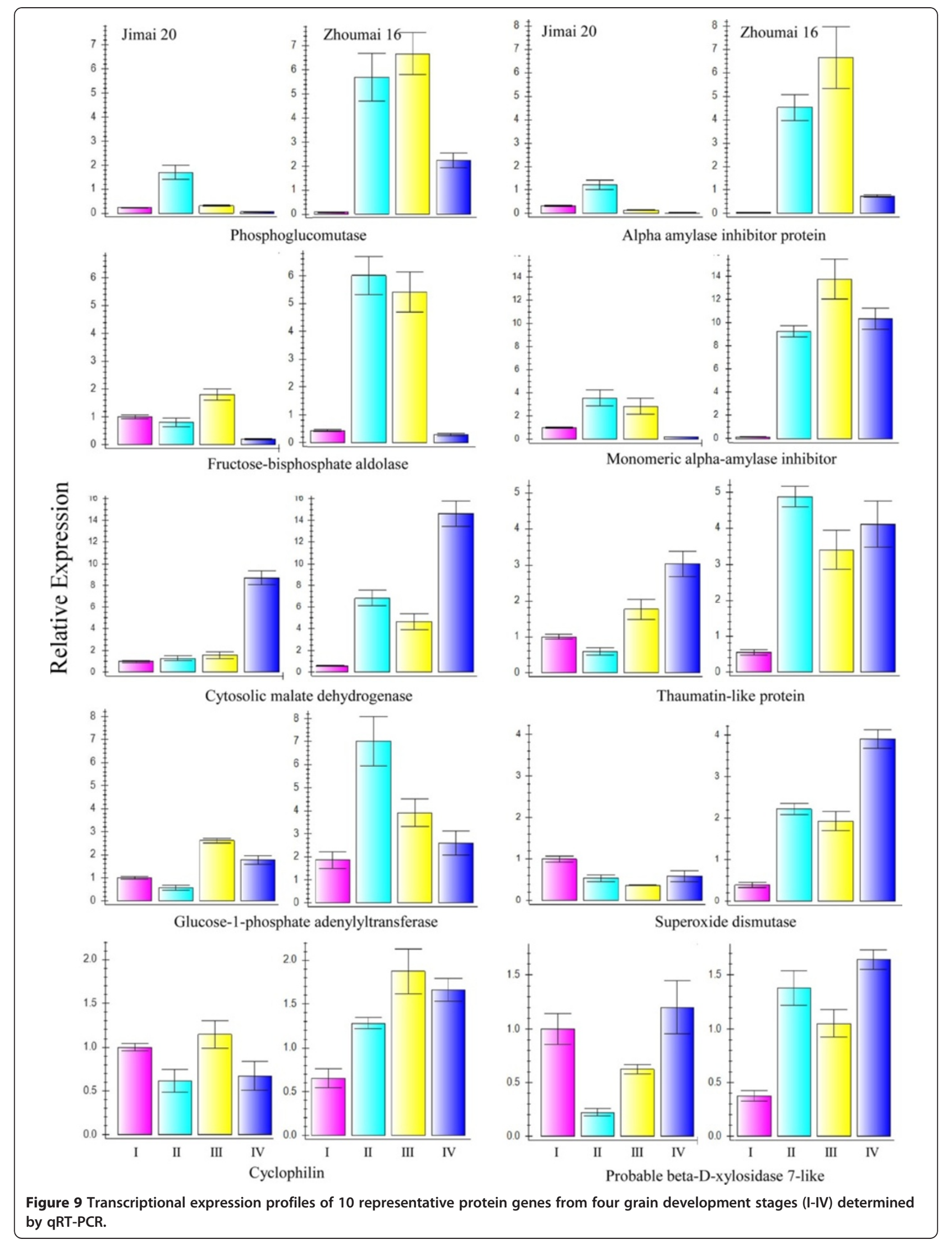


a phosphorylated protein by Pro-Q Diamond dye detection (Figure 5B), indicating its importance in glycolysis and energy provision for starch synthesis. Additionally, the expression abundance of PGM was higher in Zhoumai 16 than Jimai 20 during the early grain development stages (Figure 8 ), and this might be partly responsible for greater grain size and higher yield of Zhoumai 16 than Jimai 20.

Nucleoside diphosphate kinase (NDPK) is a ubiquitous enzyme functioning in intracellular distribution of terminal phosphate bond energy among the various nucleotides used in synthesis pathways and regulatory functions in cells $[43,44]$. Plant NDPKs are also involved in signal transduction, differentiation and development [45-47]. Our results showed that NDPK was gradually down-regulated during grain development in both wheat cultivars (Figure 9), and therefore might play an important role in signal regulation for grain development.

Glycolysis and the TCA cycle not only provide energy and intermediates for synthesis of metabolites, but their metabolite concentration gradients may also act as signals for the onset of the seed maturation phase and its regulation $[11,48]$. During the late development stages of wheat grains, the alcoholic fermentation pathway could contribute supplementary energy in anaerobic conditions due to an energy deficit from glycolysis and the TCA cycle. In addition, formate dehydrogenase showed increased expression at stage III (15 DAP) and further increased during the late grain filling and desiccation stages. This is consistent with the transition from cell growth and differentiation to starch synthesis. A switch from central carbon metabolism to alcoholic fermentation may be important for starch synthesis and accumulation during grain development [12].

\section{Starch synthesis}

Grain yield is largely determined by starch accumulation during the grain filling phase in cereals [49]. Starch biosynthesis is initiated with a substrate of ADP-glucose formed by glucose-1-phosphate adenylyltransferase (ADP-glucose pyrophosphorylase/AGPase) from glucose-l-phosphate. AGPase, located in plastids, is a major rate-limiting enzyme in seed starch biosynthesis [50]. Our results demonstrated that glucose-1-phosphate adenylyltransferase large subunits (spots 41, 44 and 45) showed the highest expression levels from 6 to 11 DPA $\left(147-252^{\circ} \mathrm{Cd}\right)$, and was then down-regulated to trace levels at grain desiccation. The expression pattern closely matched large increases in both starch content and grain weight. Plastid AGPase activity maintains higher levels from 8 to $17 \mathrm{DPA}$ and reaches a peak around $11 \mathrm{DPA}$, before declining at the later stages of endosperm development [51]. By coincidence ADPglucose pyrophosophorylase preprotein (spot 35), the precursor of AGPase in plastids, showed peak expression at $147^{\circ} \mathrm{Cd}$ (6 DPA), and was then down-regulated simultaneously in both cultivars. The increase in AGPase could result in reduced ADP-glucose pyrophosophorylase preprotein during grain development.

\section{Stress and defense}

The accumulation of starch and storage proteins was accompanied by the expression of various $\alpha$-amylase inhibitors, which are mainly located in plastids or in extracellular spaces. Alpha-amylase inhibitors play important roles in protecting starch and protein reserves in the endosperm against degradation, particularly that caused by biotic stresses such as insect attack [16,52]. We identified $11 \alpha$-amylase inhibitors (spots 94-102, 119 and 120) and all accumulated at low levels during early grain development, but were rapidly up-regulated from the starch synthesis to grain desiccation stages in both wheat cultivars, consistent with the accumulation patterns of starch and storage proteins during late grain development. Twenty-one spots were identified as $\alpha$-amylase inhibitors, predominant proteins in wheat flour [19]. Probably due to protein PTMs, the upregulation of some $\alpha$-amylase inhibitors and the downregulation of other $\alpha$-amylase inhibitors may reflect their different roles in high temperature treatments or drought stress [53].

Chitinases, mainly located extracellularly, catalyse the hydrolytic cleavage of the $\beta-1,4$-glycoside bonds present in biopolymers of $\mathrm{N}$-acetylglucosamine in chitin, a major component of cell walls in fungi. Some reports showed that transgenic wheat expressing a barley chitinase exhibit enhanced resistance against powdery mildew, leaf rust and Fusarium head blight [54-56]. In our study, five protein spots were identified as class II chitinases; all exhibited trace expression levels during early grain development, but accumulated during grain filling in both cultivars. Chitinases (spots 112, 113 and 114) with different molecular weights or isoelectric points were also shown to be phosphorylated, consistent with predictions from NetPhos 2.0 Serve and Pro-Q, using Diamond dye staining (Table 2 and Figure 5B). Many isoforms of chitinase also appeared in other plant species such as Vicia faba, Pisum sativum, Hordeum vulgare, Zea mays and Glycine max [57]. Protein modifications resulting from phosphorylation have been associated with biotic and abiotic stresses such as light, pathogen invasion, hormones, temperature stresses, and nutrient deficiencies [58-60]. Transient phosphorylated modifications of chitinases might play important roles in different stress/defense and/or detoxification mechanisms through regulation of their localization and enzyme activities.

Peroxidases are one kind of antioxidant enzymes involved in defense. Some investigations showed that 
peroxidase activity in whole wheat grains varied with development stage $[61,62]$. Plant tissues typically contain several peroxidase isoforms varying in isoelectric point values from acidic to basic [63]. In our work, three spots (spots 121, 122 and 123) were simultaneously identified as peroxidase 1 with the same molecular mass but different isoelectric points. Spots 122 and 123 were both phosphorylated (Figure 5B). Interestingly, these peroxidases exhibited different expression patterns, where spot 121 was highly abundant during phases I and II but spots 122 and 123 showed only trace expression at these stages. Spots 122 and 123 were sharply up-regulated during the following grain filling phases. Differences in peroxidase activity were also observed in developing pea seedlings [64]. This suggests that peroxidase 1 may be involved in different functions through different expression patterns or phosphorylated modification. Another antioxidant enzyme is superoxide dismutase, catalyzing the first step in the active oxygen species scavenging system [65]. This enzyme was up-regulated gradually in Zhoumai 16 whereas it maintained a stable expression level in Jimai 20 during grain development, demonstrating a significant differential expression pattern in different wheat genotypes.

\section{Storage proteins and nitrogen metabolism}

Storage proteins are major components of wheat gluten and are closely related to processing quality. In the current study five kinds of storage proteins identified during grain development included triticins, globulins, avenin-like proteins, $\gamma$-gliadins and LMW-s glutenin subunits. Four $\gamma$-gliadins (spots 74, 75, 76 and 77) were initiated from $6 \mathrm{DPA}\left(147^{\circ} \mathrm{Cd}\right)$, and then sharply upregulated at $20 \mathrm{DPA}\left(461^{\circ} \mathrm{Cd}\right)$ and remained at maximum levels until grain maturity. However, globulins (spots 70, 71, 80 and 82), avenin-like protein (spot 73) and two kinds of LMW-s glutenin subunits (spots 78 and 79) were accumulated at the later developmental stages in both wheat cultivars. The abundance of the two LMW-s glutenin subunits was higher in Jimai 20 than in Zhoumai 16 at maturity, a feature possibly related to the superior gluten quality of Jimai 20 .

Triticin, a minor storage protein in the starchy endosperm of wheat, is considered to be nutritionally rich due to the presence of a unique lysine-rich decapeptide repeat motif $[66,67]$. It is also thought to be involved in determination of flour quality because of the thiol disulfide interchange which takes place during grain maturation when subjected to dehydration stress [66]. Our results showed that the expression of triticins began at 15 DPA $\left(353^{\circ} \mathrm{Cd}\right)$ and reached a maximum that lasted until maturity in both cultivars. Previous work showed that triticins were more highly accumulated in superior quality wheat varieties than in lower quality genotypes $[15,68,69]$. In our study, triticin (spot 67) showed a slightly higher expression in Jimai 20 than in Zhoumai 16 (Figure 7). Conversely to triticin expression, triticin precursor underwent a down-regulated expression pattern in both cultivars, indicating that triticin precursor was probably consumed and converted to the accumulating triticin during grain filling. Triticin precursor also accumulated at a higher level in Jimai 20 than in Zhoumai 16 during early grain development, especially at 11 DPA $\left(252^{\circ} \mathrm{Cd}\right)$ (Figure 8). This might relate to the superior quality of Jimai 20 . One triticin spot was phosphorylated (Table 2). A recent report also identified seven protein spots from white flour of $\mathrm{cV}$ Butte 86 as triticin that had undergone post-translational modifications [19].

Generally, biosynthesis of seed storage protein is dependent on amino acid synthesis and transport of nitrogen metabolism [70]. Amino acids for protein synthesis are imported from leaves and stems by the phloem to the endosperm cavity [49]. In our study, many aminotransferases of amino-acids such as aspartate, alanine and serine as well as one putative methionine synthase were identified. These enzymes were abundant pre-storage phase from flowering to 15 DPA $\left(353^{\circ} \mathrm{Cd}\right)$ and then declined with grain maturity in both wheat cultivars.

\section{Other functional proteins}

Replication factor C (RFC), composed of one large subunit and four small subunits, is an important factor involved in DNA replication and repair mechanisms as well as cell proliferation [71]. RFC mediates genomic stability and transcriptional gene silencing in Arabidopsis [72]. They express strongly in proliferating tissues, such as shoot apical meristems (SAM) and developing grain [73]. In the present study, RFC-like protein (spot 91) accumulated in highest abundance at 6 DPA $\left(147^{\circ} \mathrm{Cd}\right)$, and then displayed down-regulated expression during subsequent grain development stages in Jimai 20, whereas it showed peak expression at 11 DPA $\left(252^{\circ} \mathrm{Cd}\right)$ in Zhoumai 16, demonstrating its expression differences in different genotypes. Tri-snRNP-specific $27 \mathrm{~K}$ protein is potentially involved in non-snRNP splicing factorsmedicated protein/protein interactions and can be phosphorylated in vitro to modulate pre-mRNA splicing at the transcriptional level [74]. We identified 4 spots as $27 \mathrm{~K}$ proteins, of which 3 (87, 88 and 90) were upregulated during grain development in both cultivars. The remaining spot (86) accumulated gradually in Zhoumai 16, whereas there was little change in Jimai 20 throughout grain development. WRKY is a relatively complex transcription factor family involved in transcriptional regulation and many other plant processes including responses to biotic and abiotic stresses [75]. In our work WRKY transcription factor was continuously up-regulated in both wheat cultivars until grain maturity, 
indicating importance during the grain development process.

\section{Conclusions}

We used proteomic approaches to characterize protein accumulation during grain development in Chinese bread wheat cultivars Jimai 20 and Zhoumai 16, which differ in gluten quality. Among 117 differentially accumulated protein spots and 82 unique proteins, there were five main expression patterns, and a considerable number of proteome expression differences occurred between the two cultivars. Some cultivar-different protein spots in Jimai 20 accumulated in higher abundance than in Zhoumai 16, such as triticin precursor and LMW-s glutenin subunit, might be related to superior gluten quality. In addition, some proteins with different isoforms in developing grains appeared to be phosphorylated, a feature that which could play important roles in wheat grain development, and possibly lead to expression differences between proteins and their mRNA. Our results provide new insights into proteome characterization during grain development in different wheat genotypes.

\section{Methods}

\section{Plant materials}

Bread wheat (Triticum aestivum L., $2 \mathrm{n}=6 \mathrm{x}=42$, AABBDD) cultivars Jimai 20 and Zhoumai 16 were planted at the experimental station of the Chinese Academy of Agricultural Science (CAAS), Beijing, during the 2010-2011 growing season (October-June). Both cultivars have almost the same growing, flowering and mature times, and their main differences are gluten quality properties, viz. Jimai 20 with superior and Zhoumai 16 with poor gluten quality [32].

Wheat plants were grown under the same natural soil conditions, fertilized (130 kg N-ha ${ }^{-1}$ ) and watered as usual. According to the method of Majoul et al. [37] grain samples were harvested during the post-anthesis period based on thermal times corresponding to the following cumulative average daily temperatures $\left({ }^{\circ} \mathrm{Cd}\right): 147^{\circ} \mathrm{Cd}(\mathrm{I}), 252^{\circ} \mathrm{Cd}$ (II), $353^{\circ} \mathrm{Cd}(\mathrm{III}), 461^{\circ} \mathrm{Cd}(\mathrm{IV})$ and $749^{\circ} \mathrm{Cd}(\mathrm{V})$ as shown as

\begin{tabular}{|c|c|c|c|}
\hline Batch/No. & Date & DPA $^{*}$ & ${ }^{\circ} \mathrm{Cd}$ \\
\hline 1 & $2011.05 .04-05.10$ & 6 & $147^{\circ} \mathrm{C}$ \\
\hline$\|$ & $2011.05 .10-05.15$ & 11 & $252^{\circ} \mathrm{C}$ \\
\hline III & 2011.05.15-05.19 & 15 & $353^{\circ} \mathrm{C}$ \\
\hline IV & $2011.05 .19-05.24$ & 20 & $461^{\circ} \mathrm{C}$ \\
\hline V & $2011.05 .24-06.05$ & 31 & $749^{\circ} \mathrm{C}$ \\
\hline
\end{tabular}

*Days post-anthesis.
Table 3. Sampled grains from three replicates were collected and stored at $-80^{\circ} \mathrm{C}$ prior to analysis.

\section{Scanning electronic microscope (SEM) studies of endosperm}

The dissection of developing grains was undertaken using a scanning electronic microscope (SEM) S-4800 FESEM (Hitachi, Japan) and the average fresh seed weight was measured. Grain samples from five stages of development were fixed in aqueous solutions containing $44.5 \%$ ethanol, $1.85 \%$ methanol and $6 \%$ glacial acetic acid for more than $24 \mathrm{~h}$, and then transferred into $70 \%$ ethanol and stored at $4{ }^{\circ} \mathrm{C}$ prior to SEM observations. Samples were dehydrated stepwise in ethanol of ascending concentrations including 70\% (15 $\mathrm{min}), 80 \%$ (15 $\mathrm{min})$, $90 \%$ (overnight) and $100 \%$. The endosperms were then treated stepwise for $15 \mathrm{~min}$ in mixtures of ethanol and isoamyl acetate with ratios 3:1, 1:1 and 1:3, and were finally soaked in isoamyl acetate for $80 \mathrm{~min}$. Finally, after critical point drying, they were observed.

\section{Protein preparation}

Grain samples of $500 \mathrm{mg}$ from the middle part of wheat ears were ground into meal in liquid nitrogen and then further ground for 7-8 min with $1 \mathrm{ml}$ of extraction buffer (400 mM sucrose, $35 \mathrm{mM}$ Tris-HCl, pH 7.5, $200 \mathrm{mM}$ EDTA, $1 \mathrm{mM}$ DTT and $1 \mathrm{mM}$ PMSF), followed by $2-3 \mathrm{~min}$ in extraction buffer (400 $\mathrm{mM}$ sucrose, 35 mM Tris- $\mathrm{HCl}$, pH 7.5, 200 mM EDTA, 400 mM Triton X-100, $1 \mathrm{mM}$ DTT and $1 \mathrm{mM}$ PMSF). Samples were shaken vigorously for $15 \mathrm{~min}$ at room temperature. After twice centrifuging for $15 \mathrm{~min}$ at $13000 \mathrm{rpm}$, the supernatants were transferred to new tubes. Protein supernatants were precipitated with $1 / 4$ volumes of cold $10 \%$ trichloroacetic acid at $-20^{\circ} \mathrm{C}$ for $2-3 \mathrm{~h}$. After centrifuging for $5 \mathrm{~min}$ at $13000 \mathrm{rpm}$, the pellets were rinsed with cold $\left(-20^{\circ} \mathrm{C}\right)$ acetone, and then thrice centrifuged at $8000 \mathrm{rpm}$ for $10 \mathrm{~min}$. After freeze-drying, the pellets were added to $300 \mu \mathrm{l}$ of solubilisation buffer (7 M urea, $2 \mathrm{M}$ thiourea, $4 \% \mathrm{CHAPS}$ ) at the room temperature for $2 \mathrm{~h}$. After removal of insoluble material by centrifuging for $10 \mathrm{~min}$ at $13000 \mathrm{rpm}$, the concentrations of protein samples were determined with a 2-D Quant Kit (Amersham Bioscience, USA).

\section{2-DE and data analysis}

Protein samples of $600 \mu \mathrm{g}$ were loaded onto Immobiline DryStrip (GE Healthcare, $18 \mathrm{~cm}, \mathrm{pH}$ 3-10 L, USA) by an Ettan $^{\mathrm{TM}}$ IPG-phor II ${ }^{\mathrm{TM}}$ system (GE Healthcare, USA). Rehydration and isoelectric focusing, equilibration of strips and SDS-PAGE were performed as described earlier [76]. 2-DE experiments were repeated three times by using protein samples independently prepared from separate seed samples. Proteins were visualized by colloidal 
Coomassie Brilliant blue (CBB) staining (R-250/G$250=4: 1$ ) and analysis was performed by ImageMaster ${ }^{\mathrm{TM}}$ 2D Platinum Software Version 5.0 (GE Healthcare) following the procedures specified in the software. Firstly, spot were detected, quantified, and background were subtracted. For spot profile analysis, the first replication 2-DE gel of 6 DPA seed samples was selected as the reference gel. All analyzed gels were matched individually to the reference gel. Then, the spots that all existed in three independent sample sets were selected and all matched spots were checked manually. At last, differentially accumulated protein spots were determined according to statistically significant changes between samples by Student's $t$-test (abundance variation at least 2-fold, $p<0.05)$. Hierarchical cluster analysis was computed to analyze differentially accumulated protein spots for protein clustering according to Pearson's distance (http:// www.broadinstitute.org/cancer/software/genepattern/).

\section{Detection of phosphorylated proteins}

Protein samples were newly extracted adding PhosSTOP Inhibitor Cocktail Tablets (Roche) after 2-DE analysis described above. Gels were stained with Pro-Q Diamond (Invitrogen) according to the manufacturer's instructions. Briefly, the gels were fixed twice in $250 \mathrm{ml}$ of immobilisation solution (50\% methanol and $10 \%$ acetic acid) for

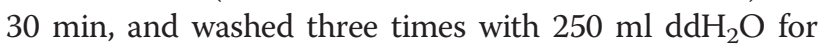
$10 \mathrm{~min}$. The gels were incubated in Pro-Q Diamond stain in darkness for $2 \mathrm{~h}$. To remove non-specific background material, the gels were thrice destained with $20 \%$ acetonitrile in $50 \mathrm{mM}$ sodium acetate $(\mathrm{pH} \mathrm{4.0)}$ for $30 \mathrm{~min}$, and

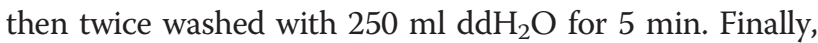
the gels were scanned on a Typhoon ${ }^{\mathrm{TM}} 9400$ scanner (GE Healthcare, USA) with a $532 \mathrm{~nm}$ excitation laser and a $580 \mathrm{~nm}$ long pass filter with a resolution of 100 microns. After fluorescent image acquisition, the gels were stained with $\mathrm{CBB}$ to visualize total proteins.

\section{Protein identification through mass spectrometry}

Differentially accumulated and phosphorylated protein spots were identified by matrix-assisted laser desorption/ ionization time-of-flight tandem mass spectrometry (MALDI-TOF/TOF-MS) according to Guo et al. [76]. Briefly, selected protein spots were excised manually from the gels and destained with $100 \mathrm{mM} \mathrm{NH}_{4} \mathrm{HCO}_{3}$ in $30 \%$ acetonitrile $(\mathrm{ACN})$ and lyophilized before digestion at $37^{\circ} \mathrm{C}$ overnight $(16 \mathrm{~h})$ with $20 \mu \mathrm{l}$ of $50 \mathrm{mM} \mathrm{NH}_{4} \mathrm{HCO}_{3}$ containing $0.01 \mathrm{mg} / \mathrm{ml}$ sequencing grade modified trypsin (Promega, Madison, WI, USA). The peptides were three times extracted with $0.1 \%$ TFA in $60 \% \mathrm{ACN}$ and tryptic peptides were then dissolved in $5 \mathrm{mg} / \mathrm{ml}$ cyano4-hydroxycinnamic acid (CHCA) in 50\% ACN and $0.1 \%$ trifluoroacetic acid (TFA). Identification of the tryptic peptides was performed using an ABI 4800 Proteomic
Analyzer MALDI-TOF/TOF-MS (Applied Biosystems/ MDS Sciex, USA). The MS together with MS/MS spectra were searched against the NCBI non-redundant viridiplantae database using softwares GPS Explorer (Applied Biosystems) and MASCOT (Matrix Science) with the following parameter settings: trypsin cleavage, two missed cleavages allowed, peptide mass tolerance set to $\pm 0.2 \mathrm{Da}$, fragment tolerance set to $\pm 0.3 \mathrm{Da}$. The proteins with Protein Score C.I. \% and Total Ion Score C.I. $\%$ above $95 \%$ were identified as credible results for MS/MS.

\section{Prediction of subcellular localization}

Subcellular locations of identified proteins were predicted using WoLF PSORT (http://wolfpsort.org/) [77], Predotar (http://urgi.versailles.inra.fr/predotar/predotar.html) [78] and UniprotKB (http://www.uniprot.org/) database programs. Protein localizations at the subcellular level were established by similar results from at least two programs.

\section{qRT-PCR analysis}

Wheat grains were ground into fine powder in liquid nitrogen and then incubated in $1 \mathrm{ml}$ TRIzol reagent (Invitrogen). Total RNA was extracted according to ther manufacturer's instructions, but adding an additional chloroform extraction. Genomic DNA was removed by digesting each sample (10 $\mu$ g of total RNA) with Rnasefree DNaseI (Promega) according to the manufacturer's instructions. After digestion, a $10 \mu \mathrm{l}$ RNA sample was dissolved for $30 \mathrm{~min}$ at the room temperature. Firststrand cDNA was synthesized in a $20 \mu \mathrm{l}$ volume containing $0.5 \mu \mathrm{l}$ AMV reverse transcriptase (Promega), $0.5 \mu \mathrm{l}$ RNase inhibitor (Promega), $1 \mu \mathrm{l}$ oligo dT primer, $2 \mu \mathrm{l}$ dNTP mixture, $4 \mu \mathrm{l} \mathrm{MgCl}_{2}(25 \mathrm{mM}), 2 \mu \mathrm{l} 10 \times$ reverse transcriptase buffer and $4 \mu \mathrm{l}$ RNA sample. The reaction mixture was incubated at $42^{\circ} \mathrm{C}$ for $60 \mathrm{~min}$.

Gene-specific primers were designed using Primer 5.0 and their specificities were checked by the melting curves of the RT-PCR products. Quantitative real-time PCR (qRT-PCR) was performed in $20 \mu \mathrm{l}$ volumes containing $10 \mu \mathrm{l} 2 \times$ SYBR $^{\circledR}$ Premix Ex Taq ${ }^{\mathrm{TM}}$ (TaKaRa), $2 \mu \mathrm{l}$ 50 -fold diluted cDNA, $0.4 \mu \mathrm{l}$ of each gene-specific pri-

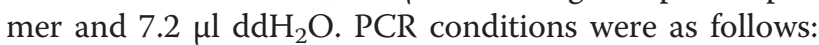
$95^{\circ} \mathrm{C}$ for $3 \mathrm{~min}, 45$ cycles of $15 \mathrm{~s}$ at $95^{\circ} \mathrm{C}, 57^{\circ} \mathrm{C}$ for $15 \mathrm{~s}$ and $72^{\circ} \mathrm{C}$ for $20 \mathrm{~s}$. Three replicates were used for each sample. Reactions were conducted in a CFX96 RealTime PCR Detection System (Bio-Rad). All data were analyzed with CFX Manager Software (Bio-Rad).

\section{Additional files}

Additional file 1: The peptide sequences of proteins during wheat grain development identified by MS/MS. 
Additional file 2: The peptide sequences of phosphorylated proteins during wheat grain development identified by MS/MS.

Additional file 3: Primer sequences used for quantitative real-time RT-PCR (qRT-PCR).

\section{Abbreviations}

2-DE: two-dimensional electrophoresis; G1P: glucose 1-phosphate; G6P: glucose 6-phosphate; MALDI-TOF/TOF-MS: matrix-assisted laser desorption/ionization time-of-flight tandem mass spectrometry; NDPK: Nucleoside diphosphate kinase; PGM: phosphoglucomutase; PTMs: post-translational modifications; qRT-PCR: real-time quantitative reverse transcriptional PCR; SEM: scanning electronic microscope.

\section{Competing interests}

This manuscript has no any financial and non-financial competing interests.

\section{Authors' contributions}

GG, LD and YX carried out all experiments and data analysis. SS, GP, LX and HY performed the preparation of CDNA, QRT-PCR and bioinformatics analyses. YY conceived the study, planned experiments, and helped draft the manuscript. All authors read and approved the final manuscript.

\section{Acknowledgements}

We are grateful to Professor Robert McIntosh from University of Sydney and Professor Rudi Appels from Murdoch University for constructive suggestions in reviewing the manuscript. This research was financially supported by grants from the Chinese Ministry of Science and Technology (2009CB118303), the National Natural Science Foundation of China (30830072, 31101145) and the National Key Projects for Transgenic Crops of China (2011ZX08009-003-004 and 2011ZX08002-004).

Received: 19 June 2012 Accepted: 16 August 2012 Published: 19 August 2012

\section{References}

1. Bietz JA, Wall JS: Wheat gluten subunits: Molecular weight determined by sodium deodecyl sulfate-polyacrylamide gel electrophoresis. J Food Sci 1972, 49:416-430.

2. Singh NK, Donovan GR, Batey IL, MacRitchie F: Use of sonication and size-exclusion high-performance liquid chromatography in the study of wheat flour proteins. I. Dissolution of total proteins in the absence of reducing agents. J Food Sci 1990, 67:150-161.

3. Wan Y, Poole RL, Huttly AK, Toscano-Underwood C, Feeney K, Welham S, Gooding MJ, Mills C, Edwards KJ, Shewry PR: Transcriptome analysis of grain development in hexaploid wheat. BMC Genomics 2008, 9:121.

4. Howarth JR, Parmar S, Jones J, Shepherd CE, Corol DI, Galster AM, Hawkins ND, Miller SJ, Baker JM, Verrier PJ: Co-ordinated expression of amino acid metabolism in response to $\mathrm{N}$ and $\mathrm{S}$ deficiency during wheat grain filling. J Exp Bot 2008, 59:3675-3689

5. Ruuska SA, Girke T, Benning C, Ohlrogge JB: Contrapuntal networks of gene expression during Arabidopsis seed filling. Plant Cell 2002, 14:1191-1206

6. Greenbaum D, Colangelo C, Williams K, Gerstein M: Comparing protein abundance and mRNA expression levels on a genomic scale. Genome Biol 2003, 4:117.

7. Zhang A, Lu Q, Yin Y, Ding S, Wen X, Lu C: Comparative proteomic analysis provides new insights into the regulation of carbon metabolism during leaf senescence of rice grown under field conditions. J Plant Physiol 2010, 167:1380-1389.

8. Li Q, Wang BC, Xu Y, Zhu YX: Systematic studies of $12 \mathrm{~S}$ seed storage protein accumulation and degradation patterns during Arabidopsis seed maturation and early seedling germination stages. J Biochem Mol Biol 2007, 40:373-381.

9. Gallardo K, Le Signor C, Vandekerckhove J, Thompson RD, Burstin J: Proteomics of Medicago truncatula seed development establishes the time frame of diverse metabolic processes related to reserve accumulation. Plant Physiol 2003, 133:664-682

10. Hajduch M, Ganapathy A, Stein JW, Thelen JJ: A systematic proteomic study of seed filling in soybean. Establishment of high-resolution two-dimensional reference maps, expression profiles, and an interactive proteome database. Plant Physiol 2005, 137:1397-1419.

11. Mechin V, Thevenot C, Le Guilloux M, Prioul JL, Damerval C: Developmental analysis of maize endosperm proteome suggests a pivotal role for pyruvate orthophosphate dikinase. Plant Physiol 2007, 143:1203-1219.

12. Xu SB, Li T, Deng ZY, Chong K, Xue Y, Wang T: Dynamic proteomic analysis reveals a switch between central carbon metabolism and alcoholic fermentation in rice filling grains. Plant Physiol 2008, 148:908-925.

13. Thelen JJ: Proteomics tools and resources for investigating protein allergens in oilseeds. Regul Toxicol Pharmacol 2009, 54(Suppl):S41-\$45.

14. Debiton C, Merlino M, Chambon C, Bancel E, Decourteix M, Planchot V, Branlard G: Analyses of albumins, globulins and amphiphilic proteins by proteomic approach give new insights on waxy wheat starch metabolism. J Cereal Sc 2011, 53:160-169.

15. Kim KH, Mostafa Kamal AH, Shin KH, Choi JS, Heo HY, Woo SH: Large-scale proteome investigation in wild relatives ( $A, B$, and $D$ genomes) of wheat. Acta Biochim Biophys Sin 2010, 42:709-716.

16. Vensel WH, Tanaka CK, Cai N, Wong JH, Buchanan BB, Hurkman WJ: Developmental changes in the metabolic protein profiles of wheat endosperm. Proteomics 2005, 5:1594-1611.

17. Dupont FM: Metabolic pathways of the wheat (Triticum aestivum) endosperm amyloplast revealed by proteomics. BMC Plant Biol 2008, 8:39.

18. Tasleem-Tahir A, Nadaud I, Girousse C, Martre P, Marion D, Branlard G: Proteomic analysis of peripheral layers during wheat (Triticum aestivum L.) grain development. Proteomics 2011, 11:371-379.

19. Dupont FM, Vensel WH, Tanaka CK, Hurkman WJ, Altenbach SB: Deciphering the complexities of the wheat flour proteome using quantitative two-dimensional electrophoresis, three proteases and tandem mass spectrometry. Proteome Sci 2011, 9:10.

20. Mamone G, De Caro S, Di Luccia A, Addeo F, Ferranti P: Proteomic-based analytical approach for the characterization of glutenin subunits in durum wheat. J Mass Spectrom 2009, 44:1709-1723.

21. Altenbach SB, Vensel WH, Dupont FM: Analysis of expressed sequence tags from a single wheat cultivar facilitates interpretation of tandem mass spectrometry data and discrimination of gamma gliadin proteins that may play different functional roles in flour. BMC Plant Biol 2010, 10:7.

22. Nadaud I, Girousse C, Debiton C, Chambon C, Bouzidi MF, Martre P, Branlard $\mathrm{G}$ : Proteomic and morphological analysis of early stages of wheat grain development. Proteomics 2010, 10:2901-2910.

23. Gao L, Wang A, Li X, Dong K, Wang K, Appels R, Ma W, Yan Y: Wheat quality related differential expressions of albumins and globulins revealed by two-dimensional difference gel electrophoresis (2-D DIGE). J Proteomics 2009, 73:279-296.

24. Lesage VS, Merlino M, Chambon C, Bouchet B, Marion D, Branlard G: Proteomes of hard and soft near-isogenic wheat lines reveal that kernel hardness is related to the amplification of a stress response during endosperm development. J Exp Bot 2012, 63:1001-1011.

25. Ge P, Ma CY, Wang SL, Gao LY, Li XH, Guo GF, Ma WJ, Yan YM: Comparative proteomic analysis of grain development in two spring wheat varieties under drought stress. Anal Bioanal Chem 2012, 402:1297-1313.

26. She M, Ye X, Yan Y, Howit C, Belgard M, Ma W: Gene networks in the synthesis and deposition of protein polymers during grain development of wheat. Func Integra Genomics 2011, 11:23-35

27. Thingholm TE, Jensen ON, Larsen MR: Analytical strategies for phosphoproteomics. Proteomics 2009, 9:1451-1468.

28. Tetlow IJ, Wait R, Lu Z, Akkasaeng R, Bowsher CG, Esposito S, Kosar-Hashemi B, Morell MK, Emes MJ: Protein phosphorylation in amyloplasts regulates starch branching enzyme activity and protein-protein interactions. Plant Cell 2004, 16:694-708.

29. Su HC, Hutchison CA, Giddings MC: Mapping phosphoproteins in Mycoplasma genitalium and Mycoplasma pneumoniae. BMC Microbiol 2007, 7:63

30. Eymann C, Becher D, Bernhardt J, Gronau K, Klutzny A, Hecker M: Dynamics of protein phosphorylation on Ser/Thr/Tyr in Bacillus subtilis. Proteomics 2007, 7:3509-3526.

31. Chitteti BR, Peng Z: Proteome and phosphoproteome dynamic change during cell dedifferentiation in Arabidopsis. Proteomics 2007, 7:1473-1500. 
32. Liu W, Zhang Y, Gao X, Wang K, Wang S, Zhang Y, He Z, Ma W, Yan Y: Comparative proteome analysis of glutenin synthesis and accumulation in developing grains between superior and poor quality bread wheat cultivars. J Sci Food Agric 2012, 92:106-115.

33. Bechtel DB, Wilson JD: Amyloplast formation and starch granule development in hard red winter wheat. Cereal Chem 2003, 80:175-183.

34. Wilson JD, Bechtel DB, Todd TC, Seib PA: Measurement of wheat starch granule size distribution using image analysis and laser diffraction technology. Tissue Cell 2006, 83:259-268.

35. Blom N, Gammeltoft S, Brunak S: Sequence and structure-based prediction of eukaryotic protein phosphorylation sites. J Mol Biol 1999, 294:1351-1362.

36. Hochwimmer $G$, Tober R, Bibars-Reiter R, Licek E, Steinborn R: Identification of two GH18 chitinase family genes and their use as targets for detection of the crayfish-plague oomycete Aphanomyces astaci. BMC Microbiol 2009, 9:184

37. Majoul T, Bancel E, Triboi E, Ben Hamida J, Branlard G: Proteomic analysis of the effect of heat stress on hexaploid wheat grain: Characterization of heat-responsive proteins from total endosperm. Proteomics 2003, 3:175-183.

38. Fernie AR, Carrari F, Sweetlove LJ: Respiratory metabolism: glycolysis, the TCA cycle and mitochondrial electron transport. Curr Opin Plant Biol 2004, 7:254-261

39. Davies EJ, Tetlow IJ, Bowsher CG, Emes MJ: Molecular and biochemical characterization of cytosolic phosphoglucomutase in wheat endosperm (Triticum aestivum L. cv. Axona). J Exp Bot 2003, 54:1351-1360

40. Fernie AR, Tauberger E, Lytovchenko A, Roessner U, Willmitzer L, Trethewey RN: Antisense repression of cytosolic phosphoglucomutase in potato (Solanum tuberosum) results in severe growth retardation, reduction in tuber number and altered carbon metabolism. Planta 2002 214:510-520.

41. Grimaud F, Rogniaux H, James MG, Myers AM, Planchot V: Proteome and phosphoproteome analysis of starch granule associated proteins from normal maize and mutants affected in starch biosynthesis. J Exp Bot 2008, 59:3395-3406.

42. Tetlow I, Liu F, Emes M: Functional interactions between starch synthases and branching enzymes of cereal endosperms. Comp Biochem Physiol, Part A: Mol Integr Physiol 2008, 150:194-195.

43. Kihara A, Saburi W, Wakuta S, Kim MH, Hamada S, Ito H, Imai R, Matsui H: Physiological and biochemical characterization of three nucleoside diphosphate kinase isozymes from rice (Oryza sativa L.). Biosci Biotechnol Biochem 2011, 75:1740-1745.

44. Shankar S, Kamath S, Chakrabarty AM: Two forms of the nucleoside diphosphate kinase of Pseudomonas aeruginosa 8830: altered specificity of nucleoside triphosphate synthesis by the cell membrane-associated form of the truncated enzyme. J Bacterio/ 1996, 178:1777-1781.

45. Choi G, Kim Jl, Hong SW, Shin B, Choi G, Blakeslee JJ, Murphy AS, Seo YW, Kim K, Koh EJ, et al: A possible role for NDPK2 in the regulation of auxin-mediated responses for plant growth and development. Plant Cell Physiol 2005, 46:1246-1254.

46. Choi G, Yi H, Lee J, Kwon YK, Soh MS, Shin B, Luka Z, Hahn TR, Song PS: Phytochrome signalling is mediated through nucleoside diphosphate kinase 2. Nature 1999, 401:610-613.

47. Tang L, Kim MD, Yang KS, Kwon SY, Kim SH, Kim JS, Yun DJ, Kwak SS, Lee HS: Enhanced tolerance of transgenic potato plants overexpressing nucleoside diphosphate kinase 2 against multiple environmental stresses. Transgenic Res 2008, 17:705-715.

48. Gutierrez L, Van Wuytswinkel O, Castelain M, Bellini C: Combined networks regulating seed maturation. Trends Plant Sci 2007, 12:294-300.

49. Emes MJ, Bowsher CG, Hedley C, Burrell MM, Scrase-Field ES, Tetlow IJ: Starch synthesis and carbon partitioning in developing endosperm. J Exp Bot 2003, 54:569-575.

50. Smidansky ED, Meyer FD, Blakeslee B, Weglarz TE, Greene TW, Giroux MJ: Expression of a modified ADP-glucose pyrophosphorylase large subunit in wheat seeds stimulates photosynthesis and carbon metabolism. Planta 2007, 225:965-976.
51. Tetlow IJ, Davies EJ, Vardy KA, Bowsher CG, Burrell MM, Emes MJ: Subcellular localization of ADP glucose pyrophosphorylase in developing wheat endosperm and analysis of the properties of a plastidial isoform. J Exp Bot 2003, 54:715-725.

52. Franco OL, Rigden DJ, Melo FR, Grossi-De-Sa MF: Plant alpha-amylase inhibitors and their interaction with insect alpha-amylases. Eur J Biochem 2002, 269:397-412

53. Yang $F$, Jørgensen AD, Li H, Søndergaard I, Finnie C, Svensson B, Jiang D, Wollenweber B, Jacobsen S: Implications of high-temperature events and water deficits on protein profiles in wheat (Triticum aestivum L. cv. Vinjett) grain. Proteomics 2011, 11:1684-1695.

54. Bliffeld M, Mundy J, Potrykus I, Futterer J: Genetic engineering of wheat for increased resistance to powdery mildew disease. Theor Appl Genet 1999, 98:1079-1086.

55. Oldach $\mathrm{KH}$, Becker D, Lorz $\mathrm{H}$ : Heterologous expression of genes mediating enhanced fungal resistance in transgenic wheat. Mol Plant Microbe Interact 2001, 14:832-838.

56. Shin S, Mackintosh CA, Lewis J, Heinen SJ, Radmer L, Dill-Macky R, Baldridge GD, Zeyen RJ, Muehlbauer GJ: Transgenic wheat expressing a barley class II chitinase gene has enhanced resistance against Fusarium graminearum. J Exp Bot 2008, 59:2371-2378.

57. Békésiová B, Hraska S, Libantová J, Moravcíková J, Matusíková I: Heavy-metal stress induced accumulation of chitinase isoforms in plants. Mol Biol Rep 2008, 35:579-588.

58. Kersten B, Agrawal GK, Durek P, Neigenfind J, Schulze W, Walther D, Rakwal R: Plant phosphoproteomics: an update. Proteomics 2009, 9:964-988

59. Kersten B, Agrawal GK, Iwahashi H, Rakwal R: Plant phosphoproteomics: a long road ahead. Proteomics 2006, 6:5517-5528.

60. Bentem S, Hirt H: Using phosphoproteomics to reveal signalling dynamics in plants. Trends Plant Sci 2007, 12:404-411.

61. Cochrane MP, Paterson L, Gould E: Changes in chalazal cell walls and in the peroxidase enzymes of the crease region during grain development in barley. J Exp Bot 2000, 51:507-520.

62. Bagatharia SB, Chanda SV: Changes in peroxidase and IAA oxidase activities during cell elongation in Phaseolus hypocotyls. Acta Physiol Planta 1998, 20:9-13.

63. Marjamaa K, Kukkola E, Lundell T, Karhunen P, Saranpaa P, Fagerstedt KV: Monolignol oxidation by xylem peroxidase isoforms of Norway spruce (Picea abies) and silver birch (Betula pendula). Tree Physiol 2006, 26:605-611.

64. Gibson DM, Liu EH: Substrate specificities of peroxidase isozymes in the developing pea seedling. Ann Bot 1978, 42:1075-1083.

65. Kaminaka H, Morita S, Tokumoto M, Masumura T, Tanaka K: Differential gene expressions of rice superoxide dismutase isoforms to oxidative and environmental stresses. Free Radical Res 1999, 31:219-225.

66. Singh NK, Donovan GR, Carpenter HC, Skerritt JH, Langridge P. Isolation and characterization of wheat triticin CDNA revealing a unique lysine-rich repetitive domain. Plant Mol Biol 1993, 22:227-237.

67. Dinesh $Y$, Nagendra KS: Wheat triticin: a potential target for nutritional quality improvement. Asian J Biotechnol 2011, 3:1-21.

68. Yahata E, Maruyama-Funatsuki W, Nishio Z, Tabiki T, Takata K, Yamamoto Y Tanida M, Saruyama $H$ : Wheat cultivar-specific proteins in grain revealed by $2-\mathrm{DE}$ and their application to cultivar identification of flour. Proteomics 2005, 5:3942-3953.

69. Garg M, Rao YS, Goyal A, Singh B: Variation in seed storage protein-triticin among diploid Triticum and Aegilops species. Biotechnol 2007, 6:444-446.

70. Hernandez-Sebastia C, Marsolais F, Saravitz C, Israel D, Dewey RE, Huber SC: Free amino acid profiles suggest a possible role for asparagine in the control of storage-product accumulation in developing seeds of low- and high-protein soybean lines. J Exp Bot 2005, 56:1951-1963.

71. Xia ST, Xiao LT, Bi DL, Zhu ZH: Arabidopsis replication factor $C$ subunit 1 plays an important role in embryogenesis. J Plant Physiol Mol Biol 2007, 33:179-187.

72. Liu Q, Wang J, Miki D, Xia R, Yu W, He J, Zheng Z, Zhu JK, Gong Z: DNA replication factor $\mathrm{C} 1$ mediates genomic stability and transcriptional gene silencing in Arabidopsis. Plant Cell 2010, 22:2336-2352. 
73. Furukawa T, Ishibashi T, Kimura S, Tanaka H, Hashimoto J, Sakaguchi K: Characterization of all the subunits of replication factor $\mathrm{C}$ from a higher plant, rice (Oryza sativa L.), and their relation to development. Plant Mol Biol 2003, 53:15-25.

74. Fetzer S, Lauber J, Will CL, Luhrmann R: The [U4/U6.U5] tri-snRNP-specific $27 \mathrm{~K}$ protein is a novel SR protein that can be phosphorylated by the snRNP-associated protein kinase. RNA 1997, 3:344-355.

75. Zhang $Y$, Wang $L$ : The WRKY transcription factor superfamily: its origin in eukaryotes and expansion in plants. BMC Evol Biol 2005, 5:1.

76. Guo GF, Ge P, Ma CY, Li XH, Lv DW, Wa SL, Ma WJ, Yan YM: Comparative proteomic analysis of salt response proteins in seedling roots of two wheat varieties. J Proteomics 2012, 75:1867-1885.

77. Horton P, Park KJ, Obayashi T, Nakai K: Protein subcellular localization prediction with WOLF PSORT. Proceedings of the 4 th Asia-Pacific Bioinformatics Conference 2006, 3:39-48.

78. Small I, Peeters N, Legeai F, Lurin C: Predotar: A tool for rapidly screening proteomes for N-terminal targeting sequences. Proteomics 2004, 4:1581-1590.

doi:10.1186/1471-2229-12-147

Cite this article as: Guo et al.: Proteome characterization of developing grains in bread wheat cultivars (Triticum aestivum L.). BMC Plant Biology 2012 12:147.

\section{Submit your next manuscript to BioMed Central and take full advantage of:}

- Convenient online submission

- Thorough peer review

- No space constraints or color figure charges

- Immediate publication on acceptance

- Inclusion in PubMed, CAS, Scopus and Google Scholar

- Research which is freely available for redistribution 